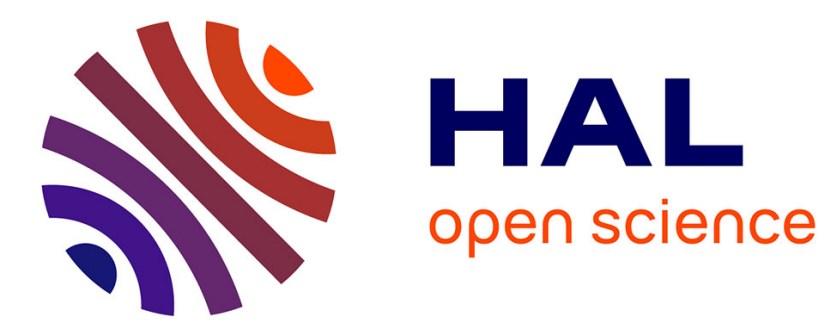

\title{
Uniting local and global output feedback controllers
}

Christophe Prieur, Andrew Teel

\section{To cite this version:}

Christophe Prieur, Andrew Teel. Uniting local and global output feedback controllers. IEEE Transactions on Automatic Control, 2011, 56 (7), pp.1636-1649. 10.1109/TAC.2010.2091436 . hal-00608429

\section{HAL Id: hal-00608429 \\ https://hal.science/hal-00608429}

Submitted on 13 Jul 2011

HAL is a multi-disciplinary open access archive for the deposit and dissemination of scientific research documents, whether they are published or not. The documents may come from teaching and research institutions in France or abroad, or from public or private research centers.
L'archive ouverte pluridisciplinaire HAL, est destinée au dépôt et à la diffusion de documents scientifiques de niveau recherche, publiés ou non, émanant des établissements d'enseignement et de recherche français ou étrangers, des laboratoires publics ou privés. 


\title{
Uniting local and global output feedback controllers
}

\author{
Christophe Prieur, and Andrew R. Teel
}

\begin{abstract}
We consider control systems for which we know two stabilizing output feedback controllers. One is globally asymptotically stabilizing, while the other one is only locally asymptotically stabilizing. We look for a composite output feedback control law that is equal to the local feedback on a neighborhood of the origin and that is globally asymptotically stabilizing. Since we want some robustness with respect to measurement noise, actuator errors and external disturbances, we need to consider hybrid output feedback controllers. Under an inputoutput-to-state stability assumption, we exhibit a solution of this uniting problem by means of a dynamic hybrid output feedback controller. Then we particularize our study to linear control systems with saturation at the input for which we know two stabilizing output feedback controllers. One is a nonlinear globally asymptotically stabilizing controller, while the other one is a high-performance linear only locally asymptotically stabilizing controller. We specify numerically tractable conditions to solve this uniting problem. Finally we illustrate our main results by means of numerical examples.
\end{abstract}

Keywords hybrid systems, global stabilization, local performance, output feedback, robustness

\section{INTRODUCTION}

\section{Background}

In nonlinear control system theory, we have now numerous tools to design (globally) asymptotically stabilizing output feedbacks, see e.g. [1], [4], [20], [24]. However, if such feedbacks give a satisfactory answer to the global asymptotic stabilization problem they are not necessarily intended to address the performance problem. As opposed to this case, for instance via linearization, one may design output feedback controllers addressing satisfactorily both the asymptotic stabilization and the performance problems but only locally. A practical example of such a framework is given by [29]. This leads us to the idea of uniting a local (optimal) output controller with a global (stabilizing) output controller, i.e. given 1) an output feedback controller $\Sigma_{0}$ able to stabilize locally while providing better performance and 2) a controller $\Sigma_{1}$ providing global asymptotic stability, we are looking for a new output feedback providing uniform global asymptotic stability for the overall system while matching exactly the local controller $\Sigma_{0}$ when the system state component is in a neighborhood of the origin.

Christophe Prieur is with Department of Automatic Control, Gipsa-lab, 961 rue de la Houille Blanche, BP 46, 38402 Grenoble Cedex, France, christophe.prieuregipsa-lab.grenoble-inp.fr. His research was supported in part by the ANR project ArHyCo, ARPEGE, contract number ANR2008 SEGI 004 01-30011459. Andrew R. Teel is with the Center for Control, Dynamical Systems \& Computation (CCDC), Department of Electrical and Computer Engineering, University of California Santa Barbara, CA 931069560; teeleece.ucsb.edu. His research was supported in part by NSF under Grant no. CCR-0311084, ECS-0622253 and by AFOSR under Grant no. AFOSR F9550-06-1-0134.
When considering only state feedbacks, this problem has been already studied and solved in [22] (cf. [30]). In that reference it is proved that, for general control systems, this uniting problem cannot be solved by considering only continuous feedbacks (best to our knowledge this problem remains open when particularizing to some classes of nonlinear systems such as the one considered in Section IV below). When using discontinuous feedbacks, we may introduce sensitivity to arbitrary small measurement noise. The design of discontinuous stabilizing controllers guaranteeing some robustness issue to measurement noise is possible with sample and hold [6], [26]. An alternative approach is found in the notion of hysteresis switching, taking advantage of the existence of a region where both output controllers $u_{0}$ and $u_{1}$ are appropriate. This suggests considering the class of hybrid controllers to solve this uniting controllers problem as done in [22] in the state feedbacks case (see also [9]). In [23] it is shown that the class of asymptotically stable hybrid systems have, under appropriate regularity properties, a robustness with respect to small measurement noise, and external disturbances. This result generalizes what has long been appreciated for continuous systems (see [7], [18]).

\section{Contribution}

Our first contribution concerns nonlinear control systems

$$
\dot{x}=f(x, u), y=h(x)
$$

where $f: \mathbb{R}^{n} \times \mathbb{R}^{m} \rightarrow \mathbb{R}^{n}$ is locally Lipschitz with $f(0,0)=0$ and $h: \mathbb{R}^{n} \rightarrow \mathbb{R}^{p}$ is continuously differentiable with $h(0)=0$. We assume that two different continuous dynamic output feedback stabilizers, $u=\alpha_{0}\left(h(x), \zeta_{0}\right), \dot{\zeta}_{0}=\varphi_{0}\left(h(x), \zeta_{0}\right)$ and $u=\alpha_{1}\left(h(x), \zeta_{1}\right), \dot{\zeta}_{1}=\varphi_{1}\left(h(x), \zeta_{1}\right)$ are given. We assume also that the nonlinear system (1) is input-output-to-state stable (IOSS) as introduced in [17] (see also [2]). Roughly speaking, this property allows us to estimate an upper bound on the magnitude of the state of the system on the basis of past input and output signals.

For linear systems this property is equivalent with the usual detectability property (see [17, Prop. 2.6]) that if the output is held equal to zero, the resulting constrained dynamics have the origin asymptotically stable. However this latter property is weaker than the IOSS for general nonlinear systems. Moreover combining the IOSS with the external stability is equivalent to the internal stability (this is the equivalence between the IOSS and the Input-to-Output Stability (IOS) with the Input-to-State Stability (ISS) property, see [27, Sec. 9]).

In the first theorem of this paper, we prove that there exists a solution of the uniting problem in terms of a hybrid output feedback controller, i.e. we state the existence of a hybrid output feedback controller which is equal to $\left(\alpha_{0}, \varphi_{0}\right)$ 
on a neighborhood of the origin and such that the system in closed loop with the hybrid feedback is globally asymptotically stable. The approach described in [22] for the state feedbacks case can not be applied, since the norm of the state is known only asymptotically. Moreover the system in closed loop with the hybrid controller proposed here has the regularity properties required in [23]. Thus we also get a robustness with respect to small measurement noise, and external disturbances, that is, for any such small perturbations, the perturbed system remains globally asymptotically stable (see Section II below for a precise statement of this result). The said class of hybrid controllers has been introduced in [10] and depends only on the output. It has a dynamic state $\zeta$, continuous dynamics $\dot{\zeta}=v(h(x), \zeta)$, if $\zeta \in \mathcal{C}$, and discrete dynamics $\zeta^{+}=w(h(x), \zeta)$, if $\zeta \in \mathcal{D}$. The system (1) will be in closed loop with the output of the hybrid controller, i.e. $u=u(h(x), \zeta)$ if $\zeta \in \mathcal{C}$.

Our second theorem makes explicit a hybrid output feedback controller solving the uniting problem under some more quantitative and weaker assumptions. These conditions are written in terms of local and global IOSS-Lyapunov functions and local and global norm-observers which exist as soon as the system (1) is IOSS, as proven in [17] (see also [15]). See Section III below for more details.

One class of nonlinear systems for which it is crucial to unite high-performance local controllers with global ones is the class of linear systems with saturation at the input. Saturation is one of the most important nonlinearities that limits control systems performance in many applications. It is known that the use of linear controllers for systems that are subject to amplitude limitation in the input may reduce the performance of the closed-loop system or even lead to instability (this is usually called the windup phenomena). Many different approaches exist in the literature for the design of static and dynamic linear anti-windup compensators (see e.g. [5], [8], [12], [21]). See also [31] where a nonlinear scheduling technique is proposed, using a switching among a family of linear gains. One way to ensure local performance with global attractivity is to unite a (optimal) linear local output feedback controller with a globally stabilizing nonlinear output feedback controller. In the third part of this work (see Section IV), we address the uniting problem of local linear stabilizer with global nonlinear stabilizer, i.e. we specify some numerically tractable conditions to design a hybrid controller which unites a prescribed global, nonlinear controller with a predesigned local, linear controller. These conditions are written in terms of a set of linear matrix inequalities (LMIs), which can be solved by efficient numerical solvers. Our result can be seen as an anti-windup result since, from a locally stabilizing controller, we build a global stabilizer. But our approach shows also how we can use output measurements to piece together arbitrary local and global output feedback controllers.

More precisely in the third part of this work, we focus on the following class of control systems

$$
\dot{x}=A x+B \operatorname{sat}(u), y=C x
$$

where $x \in \mathbb{R}^{n}, y \in \mathbb{R}^{p}, u \in \mathbb{R}^{m}, A, B$, and $C$ are matrices of appropriate dimensions, and "sat" denotes the usual (decentralized and symmetric) saturation map sat $: \mathbb{R}^{m} \rightarrow \mathbb{R}^{m}$ defined by, for all $u \in \mathbb{R}^{m}$, and for all $1 \leq i \leq m$,

$$
\operatorname{sat}(u)_{(i)}= \begin{cases}-\bar{u}_{(i)} & \text { if } u_{(i)}<-\bar{u}_{(i)}, \\ u_{(i)} & \text { if }-\bar{u}_{(i)} \leq u_{(i)} \leq \bar{u}_{(i)} \\ \bar{u}_{(i)} & \text { if } \bar{u}_{(i)}<u_{(i)} .\end{cases}
$$

In the previous, $\bar{u} \in \mathbb{R}^{m}$ is a given vector, with positive components $\bar{u}_{(i)}$, for $i=1, \ldots, m$.

We assume that two different continuous dynamic output feedback stabilizers are given: a linear one $u=C_{0} \zeta_{0}+D_{0} y$, $\dot{\zeta}_{0}=A_{0} \zeta_{0}+B_{0} y$, and a nonlinear one $u=\alpha_{1}\left(C x, \zeta_{1}\right), \dot{\zeta}_{1}=$ $\varphi_{1}\left(C x, \zeta_{1}\right)$. We know that the saturation nonlinearity at the input asks for special care, and the nonlinear system (2) in closed loop with the linear controller $u=C_{0} \zeta_{0}+D_{0} y, \dot{\zeta}_{0}=$ $A_{0} \zeta_{0}+B_{0} y$ may be unstable for some large initial conditions, even if the linear system

$$
\dot{x}=A x+B u, y=C x
$$

in closed loop with $u=C_{0} \zeta_{0}+D_{0} y, \dot{\zeta}_{0}=A_{0} \zeta_{0}+B_{0} y$ is asymptotically stable.

In Section IV below, we make explicit numerical conditions to design a hybrid output feedback controller solving the uniting problem, i.e. which is equal to the linear local controller for initial conditions in a neighborhood of the origin, and with a global basin of attraction. Thus the hybrid controller has locally the same performance as the linear controller, and is globally asymptotically stabilizing.

This paper is organized as follows. In Section II, we make precise the problem under consideration in this paper and we state the first theorem, namely the existence of a dynamic hybrid output feedback controller solving the uniting problem under appropriate hypotheses. In Section III, we make our assumptions more quantitative in order to explicit our controller. This is the second theorem of this paper. In Section IV, we particularize our study to the uniting problem of a local linear stabilizer and a global nonlinear feedback for a linear system with saturation at the input. We state some numerically tractable conditions which imply the quantitative assumptions of Section III, and thus which allow us to design a hybrid controller solving the uniting problem. We illustrate this third theorem by some simulations in Section V. Some technical proofs are contained in the appendix (see Section VI and Section VII). Section VIII contains some concluding remarks.

\section{Problem STATEMEnT And EXISTENCE OF A SOlution OF THE UNITING PROBLEM}

In this section, we define precisely the problem under consideration in this paper and we state the existence of a solution under appropriate assumptions (see Theorem 2.1 below).

Let us consider two continuous dynamic output feedback controllers $\left(\alpha_{q}, \varphi_{q}\right)$, for $q \in\{0,1\}$, where $\alpha_{0}: \mathbb{R}^{p} \times \mathbb{R}^{l_{0}} \rightarrow \mathbb{R}^{m}$, $\varphi_{0}: \mathbb{R}^{p} \times \mathbb{R}^{l_{0}} \rightarrow \mathbb{R}^{l_{0}}, \alpha_{1}: \mathbb{R}^{p} \times \mathbb{R}^{l_{1}} \rightarrow \mathbb{R}^{m}$, and $\varphi_{1}: \mathbb{R}^{p} \times \mathbb{R}^{l_{1}} \rightarrow$ $\mathbb{R}^{l_{1}}$ are continuous functions vanishing at the origin. Let us make explicit our assumption. 
Assumption 1: 1. (local dynamic controller) The origin of $\mathbb{R}^{n} \times \mathbb{R}^{l_{0}}$ is locally asymptotically stable for the system

$$
\begin{aligned}
\dot{x} & =f\left(x, \alpha_{0}\left(h(x), \zeta_{0}\right)\right), \\
\dot{\zeta}_{0} & =\varphi_{0}\left(h(x), \zeta_{0}\right) ;
\end{aligned}
$$

2. (global dynamic controller) the origin of $\mathbb{R}^{n} \times \mathbb{R}^{l_{1}}$ is globally attractive for the system

$$
\begin{aligned}
\dot{x} & =f\left(x, \alpha_{1}\left(h(x), \zeta_{1}\right)\right), \\
\dot{\zeta}_{1} & =\varphi_{1}\left(h(x), \zeta_{1}\right) ;
\end{aligned}
$$

3. (IOSS) the system (1) is input-output-to-state stable.

To be self-contained, let us recall that the system (1) is IOSS if there exist functions $\beta$ of class $\mathcal{K} \mathcal{L}$ and $\gamma$ of class $\mathcal{K}$, such that, for all ${ }^{1} u: \mathbb{R}_{\geq 0} \rightarrow \mathbb{R}^{m}$, and for all maximal trajectories $x$ of (1) with $u=u(t)$, we have, for all $t \in\left[0, t_{\text {sup }}(x)\right)$,

$$
|x(t)| \leq \max \left\{\beta(|x(0)|, t), \gamma\left(\sup _{s \in[0, t]}|y(s)|\right), \gamma\left(\sup _{s \in[0, t]}|u(s)|\right)\right\},
$$

where we denote the Euclidean norms by $|$.$| , and t_{\text {sup }}(x)$ denotes the supremum of the domain of a trajectory $x$ of (1). This notion is studied in [17]. It is proven in particular that this implies the existence of a norm-observer (this notion is used in Section III below). Weaker conditions for the existence of a norm-observer are given in [15].

In this paper we consider a dynamic hybrid output feedback controller $(C, \mathcal{D}, u, v, w)$ where, for a given integer $l$, $\mathcal{C} \subset \mathbb{R}^{l}$ and $\mathcal{D} \subset \mathbb{R}^{l}$ are closed sets, while $u: \mathbb{R}^{p} \times \mathcal{C} \rightarrow \mathbb{R}^{m}$, $v: \mathbb{R}^{p} \times \mathcal{C} \rightarrow \mathbb{R}^{l}$ and $w: \mathbb{R}^{p} \times \mathcal{D} \rightarrow \mathbb{R}^{l}$ are continuous functions. Let us make explicit our notion of trajectories of (1) in closed loop with a dynamic hybrid output feedback controller $(C, \mathcal{D}, u, v, w)$, and the notion of robust asymptotic stability. The closed-loop system lies in the class of hybrid systems as considered in e.g., [3], [19]. Here we consider the notion of trajectories as studied in [10], [11], [23]. First we recall that a set $S \subset \mathbb{R}_{\geq 0} \times \mathbb{N}$ is a compact hybrid time domain if $S=\bigcup_{j=0}^{J-1}\left[\left[t_{j}, t_{j+1}\right], j\right]$ for some finite sequence of times $0=t_{0} \leq t_{1} \leq t_{2} \ldots \leq t_{J}$. The set $S$ is a hybrid time domain if for all $(T, J) \in S, S \cap[[0, T] \times\{0,1, \ldots J\}]$ is a compact hybrid time domain.

The system (1) in closed loop with the dynamic hybrid output feedback controller $(C, \mathcal{D}, u, v, w)$ is defined as the hybrid system

$$
\left.\begin{array}{l}
\dot{x}=f(x, u(h(x), \zeta)) \\
\dot{\zeta}=v(h(x), \zeta))
\end{array}\right\} \text { if } \zeta \in \mathcal{C},
$$

Given $\left(x^{0}, \zeta^{0}\right) \in \mathbb{R}^{n} \times(\mathcal{C} \cup \mathcal{D})$, we say that $(x, \zeta)$ is a trajectory to (6) starting at $\left(x^{0}, \zeta^{0}\right)$ if $(x, \zeta)$ is defined on a hybrid time domain $\operatorname{dom}(x, \zeta)$, takes values in $\mathbb{R}^{n} \times(\mathcal{C} \cup \mathcal{D})$, has an unbounded time domain and satisfies:

(S1) for all $j \in \mathbb{N}$ and almost all $t$ such that $(t, j) \in \operatorname{dom}(x, \zeta)$, we have $\zeta(t, j) \in C$ and

$$
\left\{\begin{array}{l}
\dot{x}(t, j)=f(x(t, j), u(h(x(t, j)), \zeta(t, j))) \\
\dot{\zeta}(t, j)=v(h(x(t, j)), \zeta(t, j))
\end{array}\right.
$$

\footnotetext{
${ }^{1}$ Here and in what follows all controls are assumed to be measurable and essentially bounded functions.
}

(S2) for all $(t, j) \in \operatorname{dom}(x, \zeta)$ such that $(t, j+1) \in \operatorname{dom}(x, \zeta)$, we have $\zeta(t, j) \in \mathcal{D}$ and

$$
\left\{\begin{array}{l}
x(t, j+1)=x(t, j) \\
\zeta(t, j+1)=w(h(x(t, j)), \zeta(t, j))
\end{array}\right.
$$

(S3) $(x, \zeta)(0,0)=\left(x^{0}, \zeta^{0}\right)$.

Note that, in the previous statements (as in [10], [11], [23]), it is required that the initial conditions and the trajectories take values in $\mathbb{R}^{n} \times(C \cup \mathcal{D})$. Let us recall that the origin is globally asymptotically stable for the system (6), if

- (local stability) for each $\varepsilon>0$, there exists $\delta>0$ such that for all $\left(x^{0}, \zeta^{0}\right)$ satisfying $\left|x^{0}\right|+\left|\zeta^{0}\right| \leq \delta$ and $\left(x^{0}, \zeta^{0}\right) \in$ $\mathbb{R}^{n} \times(C \cup \mathcal{D})$, every trajectory of $(6)$ starting at $\left(x^{0}, \zeta^{0}\right)$ satisfies $|x(t, j)|+|\zeta(t, j)| \leq \varepsilon$, for all $(t, j)$ in $\operatorname{dom}(x, \zeta)$;

- (global convergence) for all $\left(x^{0}, \zeta^{0}\right) \in \mathbb{R}^{n} \times(\mathcal{C} \cup \mathcal{D})$, every trajectory of (6) starting at $\left(x^{0}, \zeta^{0}\right)$ satisfies $\lim _{t+j \rightarrow \infty}|x(t, j)|+|\zeta(t, j)|=0$.

Considering system (6) under measurement noise $\left(e_{x}, e_{\zeta}\right)$ and external disturbances $\left(d_{x}, d_{\zeta}\right)$ leads to the following system

$$
\begin{aligned}
& \left.\begin{array}{l}
\dot{x}=f\left(x, u\left(h\left(x+e_{x}\right), \zeta+e_{\zeta}\right)\right)+d_{x} \\
\left.\dot{\zeta}=v\left(h\left(x+e_{x}\right), \zeta+e_{\zeta}\right)\right)+d_{\zeta}
\end{array}\right\} \text { if } \zeta+e_{\zeta} \in C, \\
& \left.\begin{array}{l}
x^{+}=x \\
\zeta^{+}=w\left(h\left(x+e_{x}\right), \zeta+e_{\zeta}\right)
\end{array}\right\} \text { if } \zeta+e_{\zeta} \in \mathcal{D} .
\end{aligned}
$$

Now, let us recall that admissible measurement noise and admissible external disturbances are functions $e=\left(e_{x}, e_{\zeta}\right)$ and $d=\left(d_{x}, d_{\zeta}\right)$ in $\mathcal{L}_{\text {loc }}^{\infty}\left(\mathbb{R}^{n} \times \mathbb{R}^{l} \times \mathbb{R}_{\geq 0} \times \mathbb{N} ; \mathbb{R}^{n} \times \mathbb{R}^{l}\right)$ that are continuous in $(x, \zeta) \in \mathbb{R}^{n} \times \mathbb{R}^{l}$ for each $(t, j) \in \mathbb{R}_{\geq 0} \times \mathbb{N}$. We say that the origin is robustly globally asymptotically stable for the system (6), if there exists a positive definite continuous function $\rho: \mathbb{R}^{n} \times \mathbb{R}^{l} \rightarrow \mathbb{R}_{\geq 0}$ such that for all admissible measurement noise $e$ and admissible external disturbances $d$ satisfying, for all $(x, \zeta) \in \mathbb{R}^{n} \times \mathbb{R}^{l}$ and for all $(t, j) \in \mathbb{R}_{\geq 0} \times \mathbb{N}$, $|e(x, \zeta, t, j)| \leq \rho(x, \zeta)$ and $|d(x, \zeta, t, j)| \leq \rho(x, \zeta)$, the origin is globally asymptotically stable for the system (9).

Let us now define our uniting problem. We look for:

- an integer $l \geq l_{0}$, and a dynamic hybrid output feedback controller $(C, \mathcal{D}, u, v, w)$ such that $\mathcal{C}$ and $\mathcal{D}$ are closed sets, $u, v$ and $w$ are continuous functions, and such that the origin of (6) is robustly globally asymptotically stable;

- a matrix $M \in \mathbb{R}^{l_{0} \times l}$, a continuous positive definite function $\omega: \mathbb{R}^{n} \times \mathbb{R}^{l} \rightarrow \mathbb{R}_{\geq 0}$ and $r>0$ such that for all initial conditions $\left(x^{0}, \zeta^{0}\right) \in \mathbb{R}^{n} \times(\mathcal{C} \cup \mathcal{D})$, satisfying $\omega\left(x^{0}, \zeta^{0}\right) \leq r$, the trajectory of (6) starting at $\left(x^{0}, \zeta^{0}\right)$ has the hybrid time domain $[0, \infty) \times\{0\}$ and $(x(t, 0), M \zeta(t, 0))=\left(\bar{x}(t), \bar{\zeta}_{0}(t)\right)$ for some trajectory $\left(\bar{x}, \bar{\zeta}_{0}\right)$ of (4).

Let us remark that, the possible presence of Zeno solutions is considered in the framework of hybrid systems and in the definition of a global asymptotic stable equilibrium. ${ }^{2}$ However, combining the local asymptotic stability and the fact that for small initial conditions the trajectories match those of the local controller, the first $n+\ell_{0}$ components of all trajectories of (6) consist in a trajectory of (4) after a sufficiently large time.

${ }^{2}$ We recall that a Zeno trajectory is a complete trajectory with a domain which is bounded in the $t$-direction. 
In particular it excludes the presence of Zeno trajectories, and the convergence property may be rewritten as $\lim _{t \rightarrow \infty}|x(t, j)|+$ $|\zeta(t, j)|=0$. Let us remark also that the interest of the matrix $M$ lies in the fact that the dynamical state variable $\zeta \in \mathbb{R}^{l}$ is projected into the space $\mathbb{R}^{l_{0}}$ of the state variable of the local controller.

We are now in position to state our first result.

Theorem 2.1: Under Assumption 1, there exists a dynamic hybrid output feedback controller $(C, \mathcal{D}, u, v, w)$ solving the uniting problem.

Remark 2.2: Some observations are in order.

- To solve the uniting problem of two static controllers, we cannot restrict our attention to static continuous controllers (i.e. to continuous functions $x \mapsto u(x)$ ), since there exists a control system for which it is known that the uniting problem does not have any solution in this class of controllers (see [22]).

- Moreover we cannot restrict our attention to static discontinuous controllers (i.e. to discontinuous functions $x \mapsto u(x))$ to solve this uniting problem if we ask for a robust stabilization. See the example, given in [22], of an nonlinear control system, that is affine with respect to the control, for which this uniting problem does not have any solution in this class of controllers. This motivates the introduction of the hybrid controllers to solve the uniting problem.

- The controller considered in Theorem 2.1 is an output feedback since it depends only on the output $y$ and on the dynamic continuous state $\zeta$.

This theorem is an existence result. To construct a hybrid feedback solving the uniting problem, we need to make our assumptions more "quantitative". This is done in the next section, where we introduce a new set of assumptions which is valid as soon as Assumption 1 holds. We will then deduce Theorem 2.1 (see Section VI below).

\section{EXPLICIT SOLUTION OF THE UNITING PROBLEM}

In this section, we make our condition more quantitative in order to explicit a solution of our uniting problem for the nonlinear control system (1). More precisely we assume that:

Assumption 2: There exist two continuous, positive definite functions $V_{0}: \mathbb{R}^{n} \times \mathbb{R}^{l_{0}} \rightarrow \mathbb{R}_{\geq 0}$ and $V_{1}: \mathbb{R}^{n} \rightarrow \mathbb{R}_{\geq 0}$, two functions $\beta_{0}$, $\beta_{1}$ of class $\mathcal{K} \mathcal{L}$, two positive semi-definite continuous functions $\rho_{0}: \mathbb{R}^{p} \times \mathbb{R}^{l_{0}} \rightarrow \mathbb{R}_{\geq 0}$ and $\rho_{1}: \mathbb{R}^{m} \times \mathbb{R}^{p} \rightarrow \mathbb{R}_{\geq 0}$, and positive values $\varepsilon_{0 a}<\varepsilon_{0 b}$, $\varepsilon_{1 a}<\varepsilon_{1 b}$, and $\varepsilon_{2}$ such that:

1. (local dynamic controller) the origin of $\mathbb{R}^{n} \times \mathbb{R}^{l_{0}}$ is asymptotically stable for the system (4) with the basin of attraction containing $\left\{\left(x, \zeta_{0}\right), V_{0}\left(x, \zeta_{0}\right) \leq \varepsilon_{0 b}\right\}$;

2. (local norm-observer) by studying the system (4) and the system

$$
\dot{z_{0}}=-z_{0}+\rho_{0}\left(h(x), \zeta_{0}\right),
$$

for all initial conditions $\left(x^{0}, \zeta_{0}^{0}, z_{0}^{0}\right)$ in $\mathbb{R}^{n} \times \mathbb{R}^{l_{0}} \times \mathbb{R}$, we have

$$
V_{0}\left(x(t), \zeta_{0}(t)\right) \leq z_{0}(t)+\beta_{0}\left(\left|x^{0}\right|+\left|\zeta_{0}^{0}\right|+\left|z_{0}^{0}\right|, t\right),
$$

for all $t \in\left[0, t_{\sup }\left(x, \zeta_{0}, z_{0}\right)\right)$;
3. (global dynamic controller) for all trajectories of (5), we have $\limsup _{t \rightarrow \infty} V_{1}(x(t))+\left|\zeta_{1}(t)\right| \leq \varepsilon_{2}$;

4. (norm-observer for $x$ ) for each $u: \mathbb{R}_{\geq 0} \rightarrow \mathbb{R}^{m}$, by studying the system (1) and

$$
\dot{z}_{1}=-z_{1}+\rho_{1}(u, h(x))
$$

for all initial conditions $\left(x^{0}, z_{1}^{0}\right)$ in $\mathbb{R}^{n} \times \mathbb{R}$, we have

$$
V_{1}(x(t)) \leq z_{1}(t)+\beta_{1}\left(\left|x^{0}\right|+\left|z_{1}^{0}\right|, t\right),
$$

for all $t \in\left[0, t_{\text {sup }}\left(x, z_{1}\right)\right)$;

5. (estimation for the local system) for each trajectory of (4) starting from $\left\{\left(x, \zeta_{0}\right), V_{1}(x) \leq \varepsilon_{1 b}, \zeta_{0}=0\right\}$, we have $\rho_{0}\left(h(x(t)), \zeta_{0}(t)\right)<\varepsilon_{0 a}$ for all $t \geq 0$;

6. (estimation for the global system) for each trajectory of (5) starting from $\left\{\left(x, \zeta_{1}\right), V_{1}(x)+\left|\zeta_{1}\right| \leq \varepsilon_{2}\right\}$, we have $\rho_{1}\left(\alpha_{1}\left(h(x(t)), \zeta_{1}(t)\right), h(x(t))\right)<\varepsilon_{1 a}$, for all $t \geq 0$.

Let us note that, above, the assumption on the global output feedback relaxes those of Assumption 1 (compare item 3 and item 6 of Assumption 2 with respectively the attractivity and the stability as considered in item 2 of Assumption 1). Moreover we note that, in the previous assumption, item 5 is related to the stability assumption of item 1 by making explicit an estimation of the values of the $\rho_{0}$-function. Let us recall that (see [17]), under item 3 of Assumption 1, there exists an IOSS-Lyapunov function ${ }^{3}$, i.e. a $C^{\infty}$ function $V: \mathbb{R}^{n} \rightarrow \mathbb{R}_{\geq 0}$ such that

- there exist functions $v_{1}, v_{2}$ of class $\mathcal{K}_{\infty}$ satisfying $v_{1}(|x|) \leq V(x) \leq v_{2}(|x|)$, for all $x \in \mathbb{R}^{n}$,

- there exist functions $\sigma_{1}, \sigma_{2}$ of class $\mathcal{K}$ satisfying $\nabla V(x)$. $f(x, u) \leq-V(x)+\sigma_{1}(|u|)+\sigma_{2}(|h(x)|)$, for all $(x, u) \in$ $\mathbb{R}^{n} \times \mathbb{R}^{m}$

It follows that under item 3 of Assumption 1 , for all $l \in \mathbb{N}$, the function $\widetilde{V}: \mathbb{R}^{n} \times \mathbb{R}^{l} \rightarrow \mathbb{R}_{\geq 0}$ defined by $\widetilde{V}(x, \zeta)=V(x)+\frac{1}{2}|\zeta|^{2}$, satisfies, for all $(x, \zeta, u, v) \in \mathbb{R}^{n} \times \mathbb{R}^{l} \times \mathbb{R}^{m} \times \mathbb{R}^{l}$,

$$
\begin{aligned}
& \nabla \widetilde{V}(x, \zeta) \cdot(f(x, u), v) \\
& \leq-V(x)+\sigma_{1}(|u|)+\sigma_{2}(|h(x)|)+\zeta v \\
& \leq-\widetilde{V}(x, \zeta)+\sigma_{1}(|u|)+\frac{|v|^{2}}{2}+\sigma_{2}(|h(x)|)+\frac{3|\zeta|^{2}}{2}
\end{aligned}
$$

Thus $\widetilde{V}$ is an IOSS-Lyapunov function for the system

$$
\dot{x}=f(x, u), \dot{\zeta}=v
$$

where the state is $(x, \zeta)$, the input is $(u, v)$, and the output is $(h(x), \zeta)$. In other words if (1) is IOSS, then the system (14) is also IOSS. Given this IOSS-Lyapunov function $\widetilde{V}$, by defining $\rho(u, v, y, \zeta)=\sigma_{1}(|u|)+\frac{|v|^{2}}{2}+\sigma_{2}(|y|)+\frac{3|\zeta|^{2}}{2}$ for all $(u, v, y, \zeta) \in$ $\mathbb{R}^{m} \times \mathbb{R}^{l} \times \mathbb{R}^{p} \times \mathbb{R}^{l}$, the system

$$
\dot{z}=-z+\rho(u, v, h(x), \zeta)
$$

${ }^{3}$ In this paper we consider only exponential decay IOSS-Lyapunov, since the existence of an IOSS-Lyapunov function implies the existence of an exponential decay IOSS-Lyapunov function (see [17]). Under an IOSS assumption, the computation of an IOSS Lyapunov may be difficult to do. This computation is similar to the computation of a decreasing Lyapunov function from a global asymptotic stability property. IOSS Lyapunov functions are explicitly computed for the examples of Section $\mathrm{V}$ below. 
is a norm-observer, i.e. there exists a function $\beta$ of class $\mathcal{K} \mathcal{L}$, such that for each $u: \mathbb{R}_{\geq 0} \rightarrow \mathbb{R}^{m}$, and for each $v: \mathbb{R}_{\geq 0} \rightarrow \mathbb{R}^{l}$ by studying the system (14) and (15) we have $\widetilde{V}(x(t), \zeta(t)) \leq$ $z(t)+\beta\left(\left|x^{0}\right|+\left|\zeta^{0}\right|+\left|z^{0}\right|, t\right)$, for all initial conditions $\left(x^{0}, \zeta^{0}, z^{0}\right)$ in $\mathbb{R}^{n} \times \mathbb{R}^{l} \times \mathbb{R}$ and for all $t \in\left[0, t_{\text {sup }}(x, \zeta, z)\right)$.

We are now in position to state the following:

Claim 3.1: Assumption 2 holds if Assumption 1 is satisfied.

Proof of Claim 3.1. Using [17], there exists a positive definite function $V_{0}: \mathbb{R}^{n} \times \mathbb{R}^{l_{0}} \rightarrow \mathbb{R}$ (resp. $V_{1}: \mathbb{R}^{n} \rightarrow \mathbb{R}_{\geq 0}$ ), a function $\beta_{0}$ (resp. $\beta_{1}$ ) of class $\mathcal{K} \mathcal{L}$, a positive definite continuous function $\rho: \mathbb{R}^{m} \times \mathbb{R}_{0}^{l} \times \mathbb{R}^{p} \times \mathbb{R}^{p} \rightarrow \mathbb{R}_{\geq 0}$ (resp. $\rho_{1}: \mathbb{R}^{m} \times \mathbb{R}^{p} \rightarrow \mathbb{R}_{\geq 0}$ ) such that by studying the system (14) and (15) (resp. the system (1) and (12)), for each $u: \mathbb{R}_{\geq 0} \rightarrow \mathbb{R}^{m}$ and for each $v: \mathbb{R}_{>0} \rightarrow \mathbb{R}^{l_{0}}$, we have (11) (resp. (13)). Let us introduce a positive semi-definite function $\rho_{0}: \mathbb{R}^{p} \times \mathbb{R}^{l_{0}} \rightarrow$ $\mathbb{R}_{\geq 0}$ by letting $\rho_{0}\left(y, \zeta_{0}\right)=\rho\left(\alpha_{0}\left(y, \zeta_{0}\right), \varphi_{0}\left(y, \zeta_{0}\right), y, \zeta_{0}\right)$, for all $\left(y, \zeta_{0}\right) \in \mathbb{R}^{p} \times \mathbb{R}^{l_{0}}$. We get item 2 and item 4 of Assumption 2.

Let $\varepsilon_{0 b}$ be a positive real such that $\left\{V_{0}\left(x, \zeta_{0}\right) \leq \varepsilon_{0 b}\right\}$ is included in the basin of attraction of (4). This gives item 1 of Assumption 2. Let $0<\varepsilon_{0 a}<\varepsilon_{0 b}$. By item 1 of Assumption 1 , there exists $\varepsilon_{1 b}>0$ such that item 5 of Assumption 2 is satisfied. Let $0<\varepsilon_{1 a}<\varepsilon_{1 b}$. By item 2 of Assumption 1, there exists $\varepsilon_{2}>0$ such that item 6 of Assumption 2 is satisfied. Item 3 of Assumption 2 follows from item 2 of Assumption 1. This concludes the proof of Claim 3.1.

The second theorem of this paper is the design of a hybrid controller solving the uniting problem:

Theorem 3.2: Under Assumption 2, there exists a dynamic hybrid output feedback controller $(\mathcal{C}, \mathcal{D}, u, v, w)$ solving the uniting problem.

More precisely, letting $l=l_{0}+l_{1}+3$, and decomposing all $\zeta \in \mathbb{R}^{l}$ as $\zeta=\left(\zeta_{0}, \zeta_{1}, z_{0}, z_{1}, q\right)$ where $\left(\zeta_{0}, \zeta_{1}, z_{0}, z_{1}, q\right) \in \mathbb{R}^{l_{0}} \times$ $\mathbb{R}^{l_{1}} \times \mathbb{R} \times \mathbb{R} \times \mathbb{R}$, the following dynamic hybrid output feedback controller

$$
\begin{aligned}
& \mathcal{C}=\mathcal{C}_{0} \cup \mathcal{C}_{1}, \mathcal{D}=\mathcal{D}_{0} \cup \mathcal{D}_{1} \\
& u: \mathbb{R}^{p} \times \mathcal{C} \rightarrow \mathbb{R}^{m} \\
& (y, \zeta) \mapsto \alpha_{q}\left(y, \zeta_{q}\right) \\
& v: \mathbb{R}^{p} \times \mathcal{C} \rightarrow \mathbb{R}^{l} \\
& (y, \zeta) \mapsto\left((1-q) \varphi_{0}\left(y, \zeta_{0}\right), q \varphi_{1}\left(y, \zeta_{1}\right),\right. \\
& (1-q)\left(-z_{0}+\rho_{0}\left(y, \zeta_{0}\right)\right) \\
& w: \mathbb{R}^{p} \times \mathcal{D} \rightarrow \mathbb{R}^{l} \\
& \left.-z_{1}+\rho_{1}\left(\alpha_{q}\left(y, \zeta_{q}\right), y\right), 0\right) \\
& (y, \zeta) \mapsto\left(q \zeta_{0},(1-q) \zeta_{1}, 0, z_{1}, 1-q\right)
\end{aligned}
$$

where

$$
\begin{aligned}
& \mathcal{C}_{0}=\left\{\zeta: 0 \leq z_{0} \leq \varepsilon_{0 a}, 0 \leq z_{1}, \zeta_{1}=0, q=0\right\} \\
& \mathcal{C}_{1}=\left\{\zeta: z_{0}=0, \varepsilon_{1 a} \leq z_{1}, \zeta_{0}=0, q=1\right\}, \\
& \mathcal{D}_{0}=\left\{\zeta: \zeta_{1}=0, \varepsilon_{0 a} \leq z_{0}, 0 \leq z_{1}, q=0\right\} \\
& \mathcal{D}_{1}=\left\{\zeta: \zeta_{0}=0, z_{0}=0,0 \leq z_{1} \leq \varepsilon_{1 a}, q=1\right\}
\end{aligned}
$$

solves the uniting problem.

Moreover the matrix $M \in \mathbb{R}^{l_{0} \times l}$, the continuous positive definite function $\omega: \mathbb{R}^{n} \times \mathbb{R}^{l} \rightarrow \mathbb{R}_{\geq 0}$, the value $r>0$ defining the uniting problem can be chosen as $M=\left(I_{l_{0}} 0\right)$, where $I_{l_{0}}$ stands for the identity matrix in $\mathbb{R}^{l_{0} \times l_{0}}, \omega(x, \zeta)=V_{0}\left(x, \zeta_{0}\right)+$ $\left|\zeta_{1}\right|+\left|z_{0}\right|+\left|z_{1}\right|+q$, for all $(x, \zeta) \in \mathbb{R}^{n} \times \mathbb{R}^{l}$, and a value $0<r<\min \left(1, \varepsilon_{0 a}\right)$ such that for all $\left(x^{0}, \zeta_{0}^{0}\right) \in \mathbb{R}^{n} \times \mathbb{R}^{l_{0}}$ satisfying $V_{0}\left(x^{0}, \zeta_{0}^{0}\right) \leq r$, we have $\rho_{0}\left(h(x(t)), \zeta_{0}(t)\right) \leq \varepsilon_{0 a}$ where $x$ is the trajectory of (4) starting from $\left(x^{0}, \zeta_{0}^{0}\right)$.

Let us give the main ideas of the proof of Theorem 3.2. Intuitively, due to the expression of $\mathcal{C}$, for large initial conditions, the trajectories of (1) in closed loop with (16) are trajectories of (5) as long as the state variable $z_{1}$ of the global norm-observer does not reach the value $\varepsilon_{1 a}$. Due to item 3 and item 6 of Assumption 2, for sufficiently large time, the state variable $z_{1}$ becomes smaller than $\varepsilon_{1 a}$. Then the trajectory enters $\mathcal{D}_{1}$ and $\mathcal{C}_{0}$ successively. It may happen that, as the first time when we enter $\mathcal{C}_{0}$, we are not in the basin of attraction of (4). However using item 2, item 4, and item 5 of Assumption 2 , we may prove that, for sufficiently large time, $V_{0}\left(x, \zeta_{0}\right)$ is smaller than $\varepsilon_{0 b}$, and thus we eventually are in $\mathcal{C}_{0}$ and also in the basin of attraction of the local controller. Due to the expression of $\mathcal{C}_{0}$, we keep following the trajectories of (4), and, with item 1 of Assumption 2, we converge to the origin. The details of the proof need more attention and are postponed to the appendix (see Section VI). This result is applied on an example in Section V-A below.

\section{NUMERICAL COMPUTATION OF A SOLUTION OF THE UNITING PROBLEM}

In this section, we consider the linear control system (2) with saturation at the input for which we know two stabilizing output feedback controllers. One is a nonlinear globally asymptotically stabilizing controller, while the other one is a linear only locally asymptotically stabilizing controller. We suggest a numerical algorithm to compute a solution of the uniting problem. Our approach is constructive since our conditions are written in terms of LMIs.

Let us consider two continuous dynamic output feedback controllers for (2). One is assumed to be a linear output feedback:

$$
\dot{\zeta}_{0}=A_{0} \zeta_{0}+B_{0} y, u=C_{0} \zeta_{0}+D_{0} y
$$

where $\zeta_{0} \in \mathbb{R}^{l_{0}}$ is the state of the controller, and $A_{0}, B_{0}, C_{0}$ and $D_{0}$ are matrices of appropriate dimensions. The second controller is a nonlinear output feedback controller:

$$
\dot{\zeta}_{1}=\varphi_{1}\left(C x, \zeta_{1}\right), u=\alpha_{1}\left(C x, \zeta_{1}\right),
$$

where $\varphi_{1}: \mathbb{R}^{p} \times \mathbb{R}^{l_{1}} \rightarrow \mathbb{R}^{l_{1}}$, and $\alpha_{1}: \mathbb{R}^{p} \times \mathbb{R}^{l_{1}} \rightarrow \mathbb{R}^{m}$ are continuous functions vanishing at the origin. The system (2) in closed loop with a dynamic hybrid output feedback controller $(C, \mathcal{D}, u, v, w)$ is defined as the hybrid system

$$
\left.\begin{array}{rl}
\dot{x}= & A x+B \operatorname{sat}(u(C x, \zeta)) \\
\dot{\zeta}= & v(C x, \zeta)
\end{array}\right\} \text { if } \zeta \in \mathcal{C},
$$

Let us particularize Assumption 1 to the case of the control system (2) in closed loop with the output feedback laws (17) and (18): 
Assumption 3: 1. (local linear controller) The origin of $\mathbb{R}^{n} \times \mathbb{R}^{l_{0}}$ is locally asymptotically stable for the system

$$
\begin{aligned}
\dot{x} & =A x+B \operatorname{sat}\left(C_{0} \zeta_{0}+D_{0} y\right), \\
\dot{\zeta}_{0} & =A_{0} \zeta_{0}+B_{0} y
\end{aligned}
$$

2. (global nonlinear controller) the origin of $\mathbb{R}^{n} \times \mathbb{R}^{l_{1}}$ is globally attractive for the system

$$
\begin{aligned}
\dot{x} & =A x+B s a t\left(\alpha_{1}\left(C x, \zeta_{1}\right)\right), \\
\dot{\zeta}_{1} & =\varphi_{1}\left(C x, \zeta_{1}\right) .
\end{aligned}
$$

Let us note that the IOSS assumption (as given in item 3 of Assumption 1) is not explicitly needed since, as remarked in [17], the detectability of the linear system $\dot{x}=A x+B u, y=C x$ (due to item 1 of Assumption 2) implies that the linear system $\dot{x}=A x+B u, y=C x$ is IOSS which in turn implies that the system (2) is IOSS.

Therefore Assumption 3 implies Assumption 1, and we deduce from Theorem 2.1 the existence of a solution of our uniting problem as claimed in the following result.

Corollary 4.1: Under Assumption 3, there exists a dynamic hybrid output feedback controller $(C, \mathcal{D}, u, v, w)$ solving the uniting problem.

This result is an existence result. But we can prove a stronger result. Indeed under Assumption 3 we may construct a hybrid controller solving our uniting problem (see Corollary 4.5 below). To do that, we denote the usual matrix norms by $\|\cdot\|$ (without specifying the dimensions), and by $I_{n}, I_{p}, \ldots$ the identity matrix in $\mathbb{R}^{n \times n}, \mathbb{R}^{p \times p}, \ldots$ respectively, and by 0 the null matrix of appropriate dimensions. For each matrix $\mathbf{M}$, the notation $\mathbf{M}>0$ (resp. $\mathbf{M} \geq 0$ ) means that the matrix $\mathbf{M}$ is symmetric positive definite (resp. positive semi-definite). To numerically compute a solution of our uniting problem, we need the following assumption which holds whenever Assumption 3 holds. ${ }^{4}$

Assumption 4: There exist symmetric positive definite matrices $P \in \mathbb{R}^{m \times m}, \quad P_{1} \in \mathbb{R}^{n \times n}, \quad P_{0} \in \mathbb{R}^{\left(n+l_{0}\right) \times\left(n+l_{0}\right)}$ and $Q_{0} \in \mathbb{R}^{l_{0} \times l_{0}}$, a symmetric positive semidefinite matrix $N \in$ $\mathbb{R}^{\left(m+p+l_{0}\right) \times\left(m+p+l_{0}\right)}$, matrices $H \in \mathbb{R}^{m \times\left(n+l_{0}\right)}, L \in \mathbb{R}^{n \times p}$, and positive values $\varepsilon_{0 a}<\varepsilon_{0 b}$, $\varepsilon_{1 a}<\varepsilon_{1 b}$, $\varepsilon_{2}$, and $\varepsilon_{3}$ such that:

1. (local linear controller) the origin of $\mathbb{R}^{n} \times \mathbb{R}^{l_{0}}$ is asymptotically stable for the system (20) with a basin of attraction containing the set $\left\{\left(x, \zeta_{0}\right),\left(x^{\prime}, \zeta_{0}^{\prime}\right) P_{0}\left(x^{\prime}, \zeta_{0}^{\prime}\right)^{\prime} \leq \varepsilon_{3}\right\}$, the value $\left(x^{\prime}, \zeta_{0}^{\prime}\right) P_{0}\left(x^{\prime}, \zeta_{0}^{\prime}\right)^{\prime}$ is non-increasing along the trajectories of (20) starting in this set, and we have

$$
P_{1}(A+L C)+(A+L C)^{\prime} P_{1} \leq-2 P_{1} ;
$$

2. (global nonlinear controller) by defining $V_{1}: \mathbb{R}^{n} \rightarrow \mathbb{R}_{\geq 0}$ with $V_{1}(x)=x^{\prime} P_{1} x$, for all trajectories of (21), we have $\lim _{t \rightarrow \infty} V_{1}(x(t))+\left|\zeta_{1}(t)\right| \leq \varepsilon_{2}$

3. for each trajectory of (21) starting from $\left\{\left(x, \zeta_{1}\right), V_{1}(x)+\right.$ $\left.\left|\zeta_{1}\right| \leq \varepsilon_{2}\right\}$, we have

$\rho_{1}\left(\alpha_{1}\left(C x(t), \zeta_{1}(t)\right), C x(t)\right)<\varepsilon_{1 a}$, for all $t \geq 0$, where $\rho_{1}: \mathbb{R}^{m} \times \mathbb{R}^{p} \rightarrow \mathbb{R}_{\geq 0}$ is defined by $\rho_{1}(u, y)=$ $2\left[\begin{array}{c}u \\ y\end{array}\right]^{\prime}\left[\begin{array}{cc}B^{\prime} P_{1} B & 0 \\ \star & L^{\prime} P_{1} L\end{array}\right]\left[\begin{array}{l}u \\ y\end{array}\right]$, for all $(u, y) \in \mathbb{R}^{m} \times$

\footnotetext{
${ }^{4}$ For any symmetric matrix, we will denote the symmetric terms by $\star$.
}

4. the inequalities

$$
\begin{aligned}
& \varepsilon_{3} \varepsilon_{0 b} \geq \varepsilon_{1 b}, \\
& {\left[\begin{array}{cc}
\frac{1}{\varepsilon_{0 b}} P_{1} & 0 \\
\star & \frac{1}{\varepsilon_{0 b}} Q_{0}
\end{array}\right] \geq P_{0} ，} \\
& {\left[\begin{array}{cc}
\frac{\varepsilon_{0 b}}{\varepsilon_{1 b}} P_{0} & \bar{u}_{i} H_{(i)}^{\prime} \\
\star & 1
\end{array}\right] \geq 0, i=1, \ldots, m \text { ， }} \\
& N \geq\left[\begin{array}{cc}
2 B^{\prime} P_{1} B & 2 B^{\prime} P_{1} B D_{0} \\
\star & 2\left(L^{\prime} P_{1} L+D_{0}^{\prime} B^{\prime} P_{1} B D_{0}\right) \\
\star & \star
\end{array}\right. \\
& \left.\begin{array}{c}
2 B^{\prime} P_{1} B C_{0} \\
D_{0}^{\prime} B^{\prime} P_{1} B C_{0}+B_{0}^{\prime} Q_{0} \\
2 C_{0}^{\prime} B^{\prime} P_{1} B C_{0}+A_{0}^{\prime} Q_{0}+Q_{0} A_{0}+Q_{0}
\end{array}\right] \\
& {\left[\begin{array}{cc}
2 P & P(K-H) \\
\star & \frac{\varepsilon_{0} \varepsilon_{0 b}}{\varepsilon_{1 b}} P_{0}
\end{array}\right]} \\
& >\operatorname{diag}\left(I_{m}, C^{\prime}, I_{l_{0}}\right) \operatorname{Ndiag}\left(I_{m}, C, I_{l_{0}}\right)
\end{aligned}
$$

hold, where $H_{(i)}$ denotes the ith row of $H$ and $K=$ $\left(D_{0} C C_{0}\right)$.

Let us denote $\mathbf{A}=\left[\begin{array}{cc}A & 0 \\ B_{0} C & A_{0}\end{array}\right]$, and $\mathbf{B}=\left[\begin{array}{c}B \\ 0\end{array}\right]$. Let us note that, above, the assumption on the global, nonlinear output feedback relaxes those of Assumption 3 (compare item 2 and item 3 of Assumption 4 with item 2 of Assumption 3). More precisely, we may prove that Assumption 4 holds if Assumption 3 is satisfied (see Proposition 4.2 below). Moreover, the problem of the computation of the variables considered in Assumption 4 is not convex due to the products appearing in (26). However, we state below a numerical algorithm to compute a solution of the uniting problem by solving LMIs only:

Proposition 4.2: Assumption 4 holds if Assumption 3 is satisfied. Moreover the data allowing to define the hybrid controller (16) are computed solving only LMIs as follows:

Algorithm 4.3: (to compute all the variables needed to define the hybrid controller (16))

1. Compute a symmetric positive definite matrix $P_{1} \in \mathbb{R}^{n \times n}$, and a matrix $\mathcal{L} \in \mathbb{R}^{n \times p}$ solution of $P_{1} A+\mathcal{L} C+A^{\prime} P_{1}+$ $C^{\prime} L^{\prime}<-2 P_{1}$, and let $L=P_{1}^{-1} \mathcal{L}$;

2. compute a symmetric definite matrix $W_{0}$ in $\mathbb{R}^{\left(n+l_{0}\right) \times\left(n+l_{0}\right)}$, a matrix $Z \in \mathbb{R}^{m \times\left(n+l_{0}\right)}$, a diagonal positive matrix $S \in \mathbb{R}^{\left(n+l_{0}\right) \times\left(n+l_{0}\right)}$ in $\mathbb{R}^{l_{0} \times l_{0}}$ satisfying

$$
\begin{gathered}
{\left[\begin{array}{cc}
W_{0} \mathcal{A}^{\prime}+\mathcal{A} W_{0} & \mathbf{B} S-Z^{\prime} \\
\star & -2 S
\end{array}\right]<0,} \\
{\left[\begin{array}{cc}
W_{0} & W_{0} K_{(i)}^{\prime}-Z_{(i)}^{\prime} \\
\star & \bar{u}_{(i)}^{2}
\end{array}\right] \geq 0, i=1, \ldots, m,}
\end{gathered}
$$

where $\mathcal{A}=\mathbf{A}+\mathbf{B} K$, and $K_{(i)}\left(\right.$ resp. $\left.Z_{(i)}\right)$ denotes the ith row of $K$ (resp. $Z$ );

3. let $P_{0}=W_{0}^{-1}$ and $\varepsilon_{3}=1$, and compute a symmetric positive matrix $R \in \mathbb{R}^{l_{0} \times l_{0}}$, and a positive value $\varepsilon$ satisfying

$$
\left[\begin{array}{cc}
\varepsilon P_{1} & 0 \\
\star & R
\end{array}\right] \geq P_{0}
$$


Let $0<\varepsilon_{0 a}<\varepsilon_{0 b}=1 / \varepsilon$ and $Q_{0}=\varepsilon_{0 b} R$;

4. compute a matrix $H \in \mathbb{R}^{m \times\left(n+l_{0}\right)}$, and a positive value $\hat{\varepsilon}$ satisfying

$$
\left[\begin{array}{cc}
\hat{\varepsilon} P_{0} & \bar{u}_{i} H_{(i)}^{\prime} \\
\star & 1
\end{array}\right] \geq 0, i=1, \ldots, m
$$

5. compute a symmetric positive definite matrix $P \in$ $\mathbb{R}^{m \times m}$, a symmetric positive semidefinite matrix $N \in$ $\mathbb{R}^{\left(m+p+l_{0}\right) \times\left(m+p+l_{0}\right)}$ and a positive value $\widetilde{\varepsilon}$ satisfying (26) and

$$
\begin{gathered}
{\left[\begin{array}{cc}
2 P & P(K-H) \\
\star & \widetilde{\varepsilon} P_{0}
\end{array}\right]} \\
>\operatorname{diag}\left(I_{m}, C^{\prime}, I_{l_{0}}\right) \operatorname{Ndiag}\left(I_{m}, C, I_{l_{0}}\right) .
\end{gathered}
$$

Let $\varepsilon_{1 b}=\min \left(\varepsilon_{0 b} \varepsilon_{3}, \frac{\varepsilon_{0 a} \varepsilon_{0 b}}{\tilde{\varepsilon}}, \frac{\varepsilon_{0 b}}{\hat{\varepsilon}}\right)$ and $0<\varepsilon_{1 a}<\varepsilon_{1 b}$.

The proof of this result is postponed until Section VII. Note that in the proof of Proposition 4.2, the basin of attraction of (20) is estimated. To do that, we used the modified condition of [8], but other approaches are possible (consider e.g., [13], [14]).

With Assumption 4 we may design a dynamic hybrid output feedback controller $(C, \mathcal{D}, u, v, w)$ solving the uniting problem. This is our third theorem.

Theorem 4.4: Under Assumption 4, letting $l=l_{0}+l_{1}+3$, $\alpha_{0}\left(y, \zeta_{0}\right)=C_{0} \zeta_{0}+D_{0} y, \varphi_{0}\left(y, \zeta_{0}\right)=A_{0} \zeta_{0}+B_{0} y$ and $\rho_{0}\left(y, \zeta_{0}\right)=$ $\left(\operatorname{sat}\left(\alpha_{0}\left(y, \zeta_{0}\right)\right)^{\prime}-\alpha_{0}\left(y, \zeta_{0}\right)^{\prime}, \quad y^{\prime}, \quad \zeta_{0}^{\prime}\right) N\left(\operatorname{sat}\left(\alpha_{0}\left(y, \zeta_{0}\right)\right)^{\prime}-\right.$ $\left.\alpha_{0}\left(y, \zeta_{0}\right)^{\prime}, y^{\prime}, \zeta_{0}^{\prime}\right)^{\prime}$, for all $\left(y, \zeta_{0}\right) \in \mathbb{R}^{p} \times \mathbb{R}^{l_{0}}$, the dynamic hybrid output feedback controller $(C, \mathcal{D}, u, v, w)$ defined by (16) solves the uniting problem.

Combining Proposition 4.2 and Theorem 4.4, we readily deduce:

Corollary 4.5: Under Assumption 3, the dynamic hybrid output feedback controller (16) solves the uniting problem and is defined solving only LMIs by following Algorithm 4.3 and by defining $\rho_{1}(u, y)=2\left(u^{\prime}, y^{\prime}\right) \operatorname{diag}\left(B^{\prime} P_{1} B, L^{\prime} P_{1} L\right)\left(u^{\prime}, y^{\prime}\right)^{\prime}$, for all $(u, y) \in \mathbb{R}^{m} \times \mathbb{R}^{p}$ and $l, \alpha_{0}, \varphi_{0}$ and $\rho_{0}$ as in Theorem 4.4.

This latter result is applied on one example in Section V-B below. To prove Theorem 4.4 we first state that Assumption 2 holds if Assumption 4 is satisfied. Then we use Theorem 3.2 to prove that the hybrid controller (16) solves the uniting problem. The complete proof in postponed until the appendix (see Section VII below).

\section{NUMERICAL SIMULATIONS}

In this section some numerical simulations are performed to illustrate the main results of this paper. It is first considered an example borrowed from the literature and Theorem 3.2 is applied. Then we apply Corollary 4.5 on an example in Section V-B. ${ }^{5}$

\section{A. An example borrowed from the literature}

Let us consider the following SISO system (see [1])

$$
\dot{x}_{1}=-x_{1}+\left(u-x_{2}\right) x_{1}^{2}, \quad \dot{x}_{2}=-x_{2}+x_{1}^{2}, \quad y=x_{1}
$$

where $x=\left(x_{1}, x_{2}\right)$ is the plant state, $y$ stands for the output, and $u$ stands for the input. Let us rewrite this system as (1)

\footnotetext{
${ }^{5}$ The simulation codes can be downloaded from www.gipsa-lab.fr/ christophe.prieur/Codes/code-uniting.zip.
}

with obvious definitions of $f: \mathbb{R}^{2} \times \mathbb{R} \rightarrow \mathbb{R}^{2}$ and $h: \mathbb{R}^{2} \rightarrow \mathbb{R}$. An observer for $x_{2}$ is $\dot{\hat{x}}_{2}=-\hat{x}_{2}+y^{2}$. Moreover, given any trajectory of (33), we compute, for all $t \in\left[0, t_{\text {sup }}(x)\right)$,

$$
\begin{aligned}
\left|x_{1}(t)\right|+\left|x_{2}(t)\right| \leq & |y(t)|+\left|x_{2}(t)-\hat{x}_{2}(t)\right|+\left|\hat{x}_{2}(t)\right| \\
\leq & |y(t)|+\left|x_{2}(0)-\hat{x}_{2}(0)\right| e^{-t} \\
& +\sup _{s \in[0, t]}|y(s)|^{2}+\left|\hat{x}_{2}(0)\right| e^{-t} \\
\leq & \left|x_{2}(0)\right| e^{-t}+\sup _{s \in[0, t]}|y(s)|^{2}
\end{aligned}
$$

if moreover $\hat{x}_{2}(0)=0$. Thus the system (33) is IOSS.

Now, for the local controller, let us pick $\alpha_{0}(y)=0$, for all $y$ in $\mathbb{R}$. By considering the linearization around the origin, it can be checked that the origin of $\mathbb{R}^{2}$ is locally asymptotically stable for system (4).

A global dynamic controller is computed in [1]. More precisely, let $\alpha_{1}: \mathbb{R} \rightarrow \mathbb{R}$ be defined by $\alpha_{1}\left(\zeta_{1}\right)=\zeta_{1}$ where $\zeta_{1}$ is a dynamical state with the following dynamics (see [1]):

$$
\dot{\zeta}_{1}=-\zeta_{1}+y^{2}-2 y^{5}
$$

System (33) in closed loop with this controller is (5) with an obvious definition of $\varphi_{1}: \mathbb{R} \times \mathbb{R} \rightarrow \mathbb{R}$. Therefore Assumption 1 holds and, applying Theorem 2.1, there exists a dynamic hybrid output feedback controller $(C, \mathcal{D}, u, v, w)$ solving the uniting problem.

To compute such a controller, let us consider Assumption 2. Let $V_{0}: \mathbb{R}^{2} \rightarrow \mathbb{R}_{>0}$ be the continuous, positive definite function defined by $V_{0}\left(x_{1}, x_{2}\right)=\frac{1}{2}\left(x_{1}^{2}+x_{2}^{2}\right)$. Simple computations give, for all $(x, u)$ in $\mathbb{R}^{2} \times \mathbb{R}$,

$$
\begin{aligned}
\nabla V_{0}(x) \cdot f(x, u) & =-x_{1}^{2}-x_{1}^{3} x_{2}-x_{2}^{2}+x_{2} x_{1}^{2}+u x_{1}^{3}, \\
& \leq-x_{1}^{2}-\frac{1}{2} x_{2}^{2}+\frac{3}{2} x_{1}^{6}+x_{1}^{4}+\frac{1}{2} u^{2},
\end{aligned}
$$

and thus $\nabla V_{0}(x) \cdot f\left(x, \alpha_{0}(h(x))\right) \leq-V_{0}(x)+\rho_{0}(y)$, where $\rho_{0}(y)=\frac{3}{2} y^{6}+y^{4}$, for all $y$ in $\mathbb{R}$. Therefore by letting, for all $(s, t)$ in $\mathbb{R}_{>0}^{2}, \beta_{0}(s, t)=3 \max \left\{s, s^{2}\right\} e^{-t}$, item 2 of Assumption 2 holds. To compute an estimation of the basin of attraction of system (4) we may check, using (35), that, for all $\left(x_{1}, x_{2}\right) \neq 0$ such that $V_{0}\left(x_{1}, x_{2}\right) \leq \frac{1}{3}$, it holds $\nabla V_{0}(x) \cdot f\left(x, \alpha_{0}(h(x))\right)<0$. Therefore by letting $\varepsilon_{0 b}=\frac{1}{3}$, item 1 of Assumption 2 holds. Pick $\varepsilon_{0 a}=\frac{1}{4}$. From (35), by letting $\rho_{1}(u, y)=\frac{1}{2} u^{2}+y^{4}+\frac{3}{2} y^{6}$, we get that (12) is a norm-observer for $x$, as consider in item 4 of Assumption 2. By letting $V_{1}=V_{0}$ and $\varepsilon_{1 b}=\frac{1}{6}$, and using that all $x_{1}$ such that $x_{1}^{2} \leq \frac{1}{3}$ satisfies $\frac{3}{2} x_{1}^{6}+x_{1}^{4} \leq \varepsilon_{0 a}$, we get that item 5 of Assumption 2 holds. Let $\varepsilon_{1 a}=\frac{1}{7}$.

Since the origin of $\mathbb{R}^{3}$ is globally asymptotically stable for the system (5), item 6 of Assumption 2 holds for a sufficiently small positive value $\varepsilon_{2}$. Therefore Assumption 2 is satisfied.

By applying Theorem 3.2, the dynamic hybrid output feedback controller (16) solves the uniting problem. To check that the origin is a global asymptotic stable equilibrium, let us plot the trajectory of (6) starting from $x^{0}=(1,-1)^{\prime}, \zeta_{1}^{0}=0$, $z_{0}^{0}=0, z_{1}^{0}=0$, and $q^{0}=0$. We check on Figure 1 (top) that the trajectory converges to the origin. The time evolution of the control values are given in Figure 1 (down). First the local controller is used up to the time instant 0.12 and then the global controller. After $t=2.3$ the local controller is used. 

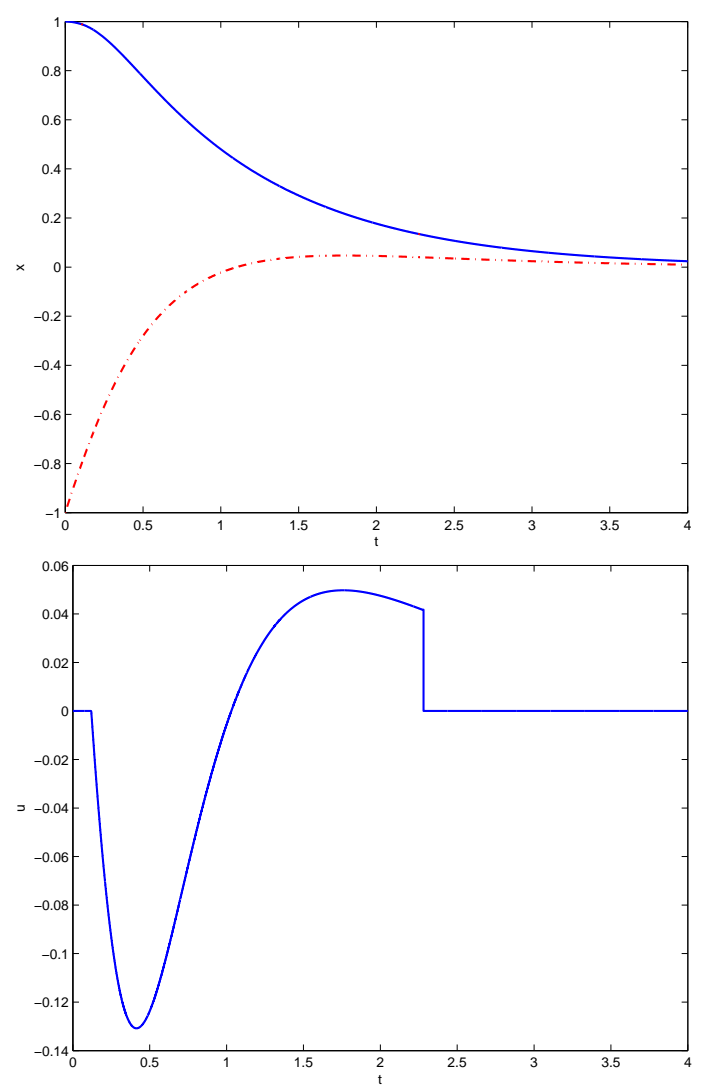

Fig. 1. Top: time evolution of $x_{1}$ (in plain line) and of $x_{2}$ (in dashed line) of the system in closed loop with the hybrid controller. Down: time evolution of $u$

\section{B. An academic example}

Before introducing the example under consideration in this section, let us recall that Corollary 4.5 can be seen as an antiwindup result since, from a locally stabilizing controller, we build a global stabilizer. Thus it may be important to maximize the size of the region where the local controller is used. For this aim, due to the uniting problem, we have to maximize the value $\varepsilon_{0 a}$. To do this, it is possible to consider some convex optimization problems derived from Algorithm 4.3. First, at step 2 of Algorithm 4.3, maximizing the estimate of the basin of attraction of (20) can be accomplished by solving the following convex optimization problem:

$$
\min \mu \text { subject to (28), (29) and }\left[\begin{array}{cc}
\mu I_{n+l_{0}} & I_{n+l_{0}} \\
\star & W_{0}
\end{array}\right]>0 .
$$

Also at step 3 of Algorithm 4.3, it is possible to maximize the value $\varepsilon_{0 a}$ by solving the convex optimization problem: min $\varepsilon$ subject to (30). These optimization objectives have been considered in the next example.

To illustrate Corollary 4.5, let us consider the twodimensional system (2), with $A=\left[\begin{array}{cc}0 & 1 \\ 0 & -0.1\end{array}\right], B=\left[\begin{array}{l}0 \\ 1\end{array}\right]$, and $C=\left[\begin{array}{ll}1 & 0\end{array}\right]$ and sat is the saturation function with level equal to 10 . The equations model a positioning system where the position $x_{1}$ is assumed to be measured, and the force which is applied on the system may saturate. The speed $x_{2}$ is subject to friction.

For the local controller we consider the following linear controller:

$$
\dot{\zeta}_{0}=A_{0} \zeta_{0}+B_{0} y, u=C_{0} \zeta_{0}+D_{0} y
$$

with $A_{0}=\left[\begin{array}{cc}-14 & 0 \\ 1 & 0\end{array}\right], B_{0}=\left[\begin{array}{c}16 \\ 0\end{array}\right], C_{0}=\left[\begin{array}{ll}7.5 & -0.625\end{array}\right]$ and $D_{0}=-10$. We easily check that the origin is asymptotically stable for the system (2) in closed-loop with (36) linearized around the origin. However the origin of the nonlinear closed-loop system is not globally asymptotically stable (consider e.g., the trajectory starting from $\left(x^{0 \prime}, \zeta_{0}^{0 \prime}\right)=$ $[10,10,10,10]$ which diverges as the time goes to the infinity).

For the second controller, consider the following static position feedback: $\alpha_{1}(y)=K y$ with $K=-0.1$. Using the positive definite function $V: \mathbb{R}^{2} \rightarrow \mathbb{R}_{\geq 0}$ defined by $V\left(x_{1}, x_{2}\right)=$ $\frac{1}{2} x_{2}^{2}-\int_{0}^{x_{1}} \operatorname{sat}(K s) d s$, for all $\left(x_{1}, x_{2}\right) \in \mathbb{R}^{2}$, and the Invariance Principle (see [16]), we may check that the origin of the system (2) in closed loop with $\alpha_{1}$ is globally asymptotically stable, but may induce large intermediate values (consider e.g., the trajectory starting from $x^{0 \prime}=[10,10]$ ).

Therefore Assumption 3 holds (recall that, as remarked after the statement of this assumption, the IOSS does need to be explicitely stated). Thus the uniting problem is solved by applying Corollary 4.5, and the hybrid controller (16) is computed by following Algorithm 4.3. It gives the following quantities

$$
\begin{gathered}
P_{1}=\left[\begin{array}{cc}
1.9655 & -0.7578 \\
\star & 0.6014
\end{array}\right], L=\left[\begin{array}{c}
-3.0951 \\
-4.7740
\end{array}\right], \\
{\left[\begin{array}{c}
\varepsilon_{0 a} \\
\varepsilon_{1 a}
\end{array}\right]=\left[\begin{array}{ccc}
0.5645 \\
1.5033 \times 10^{-4}
\end{array}\right]} \\
N=\left[\begin{array}{cccc}
55.0216 & -275.94 & 206.94 & -17.250 \\
\star & 12069 & -8673.7 & 934.16 \\
\star & \star & 7275.6 & -164.14 \\
\star & \star & \star & 347.19
\end{array}\right] .
\end{gathered}
$$

In particular, $x \mapsto x^{\prime} P_{1} x$ is an IOSS Lyapunov function for (2). Consider system (2) in closed loop with the dynamic hybrid output feedback controller (16). Let us first consider the following initial condition: $x^{0 \prime}=[0 ; 0.05 ; 0 ; 0], q^{0}=1$, $z_{1}{ }^{0}=0.05$, and $z_{0}^{0}=0.05$. We note on Figure 2 (down) that we start using the global controller until the time $t=60$. After this time instant we use the local controller and see that the trajectory tends to the origin (see Figure 2, top). We see on Figure 2 (down) that this switch is due to the fact that the value of $z_{1}$ becomes lower than $\varepsilon_{1 a}$ at the switching time.

Now we consider the initial condition $x^{0 \prime}=[0,0.05,0,0]$, $q^{0}=0, z_{1}^{0}=0.05$, and $z_{0}^{0}=0.05$. We note on Figure 4 (top) that the local controller is used until the time $t=0.84$, where $z_{0}(0.84)=0.5668>\varepsilon_{0 a}$. Thus the global controller is used. We eventually switch to the local controller (after the time $t=39$ ) (see Figure 3, down) and the trajectory converges to the origin (see Figure 3, top).

With Corollary 4.5, we also get a robustness with respect to small measurement noise. To illustrate this on numerical simulations, let us consider again the initial condition $x^{0 \prime}=$ $[0 ; 0.05 ; 0 ; 0], q^{0}=0, z_{1}^{0}=0.05$, and $z_{0}^{0}=0.05$, and an additive small noise in the output. This noise is a uniform distribution between -0.01 and 0.01 . The results are shown in Figure 5. We note that the switching sequence in not the same as the unperturbed case (compare Figure 5 (down) with 

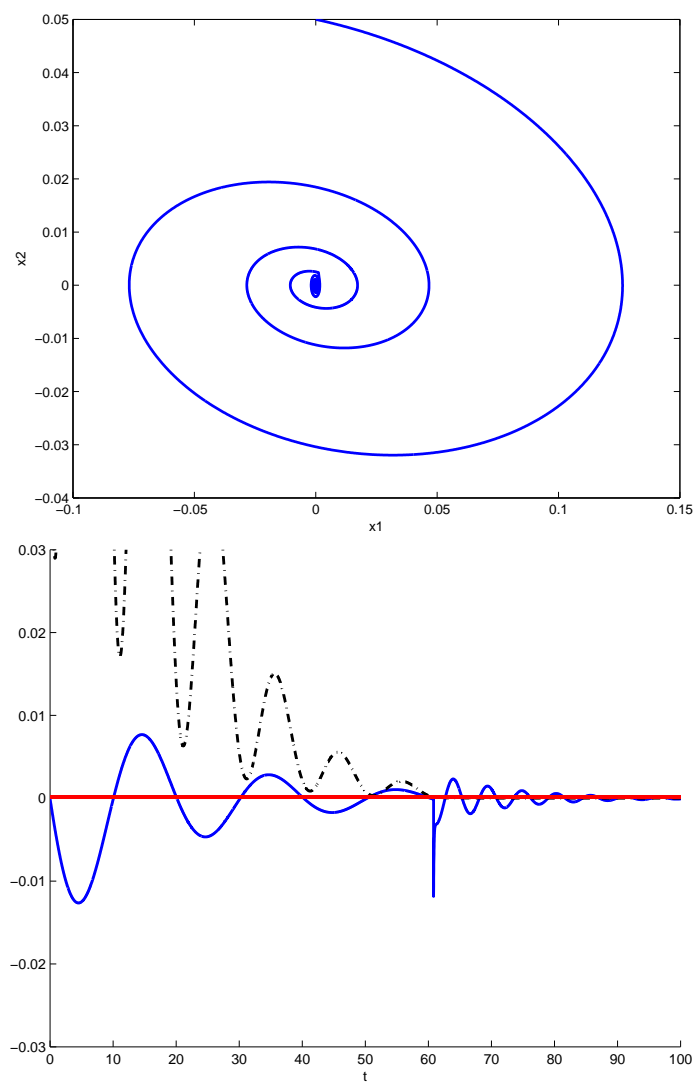

Fig. 2. Top: time evolution of $\left(x_{1}, x_{2}\right)$ of the system in closed loop with the hybrid controller. Down: time evolution of $u$ (in plain line) and of $z_{1}$ (in dashdotted line), the value $\varepsilon_{1 a}$ is given by the horizontal line.
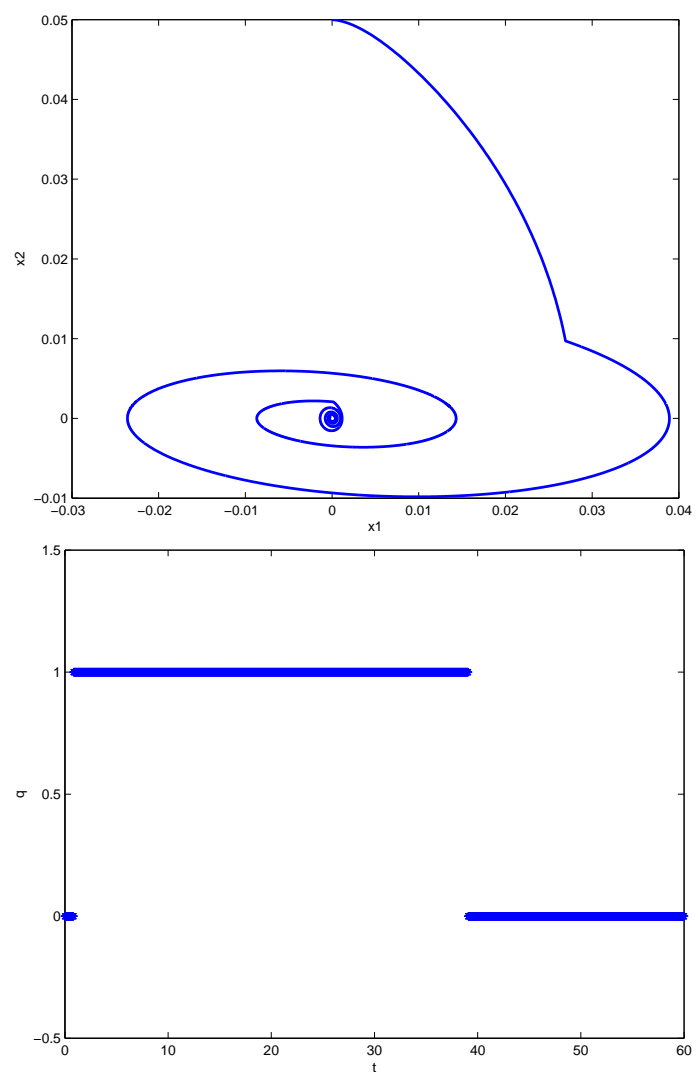

Fig. 3. Top: time evolution of $\left(x_{1}, x_{2}\right)$ of the system in closed loop with the hybrid controller. Down: time evolution of $q$.
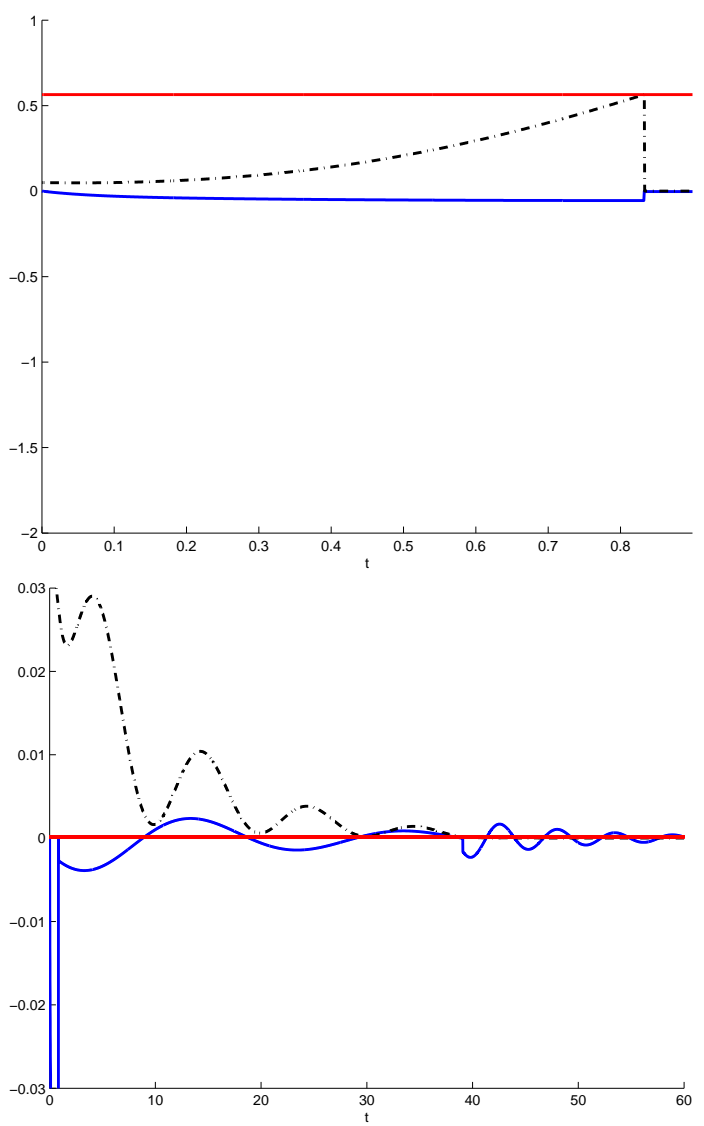

Fig. 4. Top: time evolution of $u$ (in plain line) and of $z_{0}$ (in dashdotted line), the value $\varepsilon_{0 a}$ is given by the horizontal line before the first switching time. Down: time evolution of $u$ (in plain line) and of $z_{1}$ (in dashdotted line), the value $\varepsilon_{1 a}$ is given by the horizontal line.

Figure 3 (down)) but the state is practically stabilized to the origin (see Figure 5 (top)). Due to the perturbations, even for large time, we may use the global controller (see Figure 5 (down)). Note finally that we see on Figure 6 (top) that for all initial conditions $\left(x^{0}, \zeta^{0}, z_{0}^{0}, z_{1}^{0}\right)$ with Euclidian norm less that 0.01 , only the linear controller (36) is used. This is an illustration of the second point of the uniting problem (see before Theorem 2.1). That region is smaller than the basin of attraction of the linear controller (36). This is due to the conservatism introduced in Algorithm 4.3 where we need to nest together different ellipsoidal domains. See as example, on Figure 6 (down), the projection of both ellipsoids involved in (30) on the last two coordinates $\left(x_{3}, x_{4}\right)$-plane. For this numerical example the ellipsoidal domains have slightly different profiles.

\section{Proof of Theorem 2.1 AND TheOrEM 3.2}

Let Assumption 2 hold, and consider the system (1) in closed loop with the hybrid feedback (16). Define the matrix $M \in \mathbb{R}^{l_{0} \times l}$, the continuous positive definite function $\omega$ and the value $r>0$ as introduced in Theorem 3.2.

One important remark for our proof is that, as also noted in [17], the system (10) is input-to-state stable (ISS) for the input $\left(h(x), \zeta_{0}\right)$. Similarly the system (12) is ISS for the input $(u, h(x))$. 

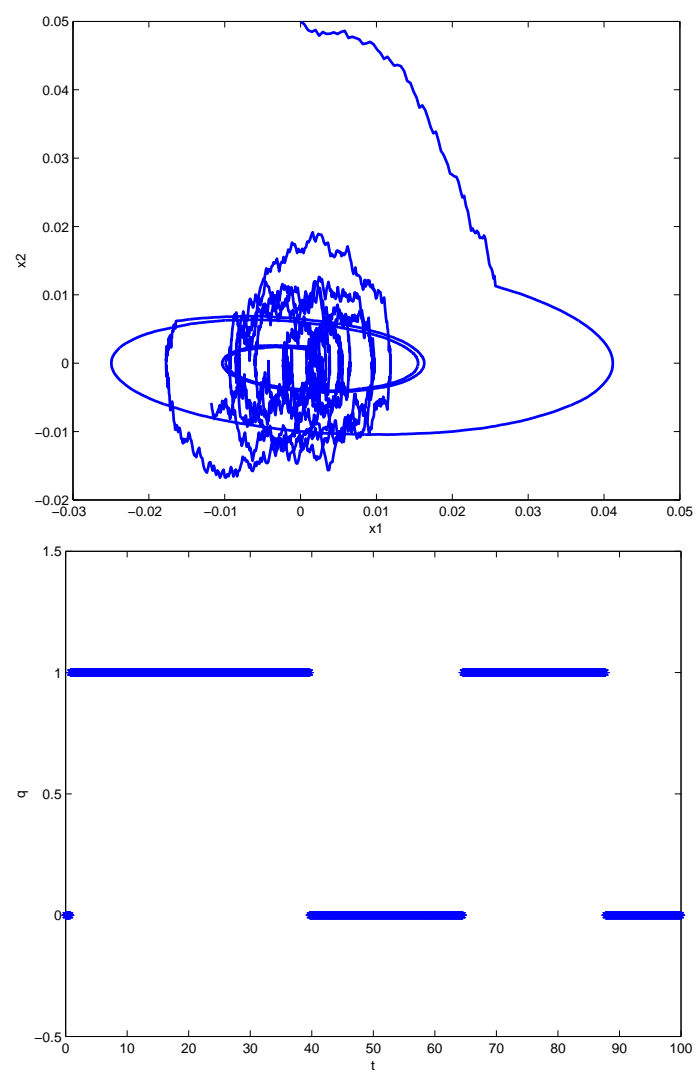

Fig. 5. Top: time evolution of $x_{1}$ (in plain line) and of $x_{2}$ (in dotted line) of the system in closed loop with the hybrid controller corrupted with noise. Down: time evolution of $q$.

Proof of Theorem 3.2. Let us prove that the origin is globally asymptotically stable for the system (6). First, let us note that the sets $\mathcal{C}_{q}, \mathcal{D}_{q}$ are closed, the function

$(x, \zeta) \mapsto\left(f(x, u(h(x), \zeta), v(h(x), \zeta))\right.$ is continuous on $\mathbb{R}^{n} \times \mathcal{C}_{q}$, and the function $(x, \zeta) \mapsto w(h(x), \zeta)$ is continuous on $\mathbb{R}^{n} \times \mathcal{D}_{q}$. Moreover, since $\rho_{0}$ and $\rho_{1}$ are positive semi-definite functions, when flowing from the boundary of $\mathcal{C}$, we enter $C$. More precisely, recall that, in $\mathcal{C}, \dot{q}=0$, and $\dot{z}_{q} \geq 0$ when $z_{q}=0$, for all $q \in\{0,1\}$, then, for all $(y, \zeta) \in \mathbb{R}^{p} \times(\partial \mathcal{C} \backslash \mathcal{D})$, we have $\{v(y, \zeta)\} \cap T_{C}(\zeta) \neq \emptyset$, where $T_{C}(\zeta)$ stands for the tangent cone to $C$ at $\zeta$. Also, for all $(y, \zeta) \in \mathbb{R}^{p} \times \mathcal{D}$, we have $w(y, \zeta) \in \mathcal{C} \cup \mathcal{D}$. Therefore, the viability conditions of [11, Prop. 2.1] are satisfied and, for all initial conditions $\left(x^{0}, \zeta^{0}\right) \in \mathbb{R}^{n} \times(\mathcal{D} \cup \mathcal{D})$, there exists a maximal trajectory of (6) starting at $\left(x^{0}, \zeta^{0}\right)$. Moreover each maximal trajectory to (6) either has an unbounded hybrid time domain or eventually leaves any compact subset of $\mathbb{R}^{n} \times \mathbb{R}^{l}$.

Let us prove the local stability property. Let $0<\varepsilon<\varepsilon_{0 a}$. Let $0<\delta<\min \left(1, \varepsilon_{0 a}\right)$ be such that, for all $\left(x^{0}, \zeta_{0}^{0}, z_{0}^{0}, z_{1}^{0}\right)$ satisfying $V_{0}\left(x^{0}, \zeta_{0}^{0}\right)+\left|z_{0}^{0}\right|+\left|z_{1}^{0}\right| \leq \delta$, the trajectory of (4), (10) and (12) with $u(t)=\alpha_{0}\left(h(x(t)), \zeta_{0}(t)\right)$, starting at $\left(x^{0}, \zeta_{0}^{0}\right)$, satisfies, for all $t \geq 0$,

$$
|x(t)|+\left|\zeta_{0}(t)\right|+\left|z_{0}(t)\right|+\left|z_{1}(t)\right| \leq \varepsilon .
$$

Such $\delta>0$ exists by item 1 of Assumption 2, and since the systems (10) and (12) (with $u=\alpha_{0}\left(h(x), \zeta_{0}\right)$ ) are ISS.

Now consider a trajectory $(x, \zeta)$ of (6) with initial condition $\left(x^{0}, \zeta^{0}\right) \in \mathbb{R}^{n} \times(\mathcal{C} \cup \mathcal{D})$ such that $V_{0}\left(x^{0}, \zeta_{0}^{0}\right)+\left|z_{0}^{0}\right|+\left|z_{1}^{0}\right|+q^{0} \leq$
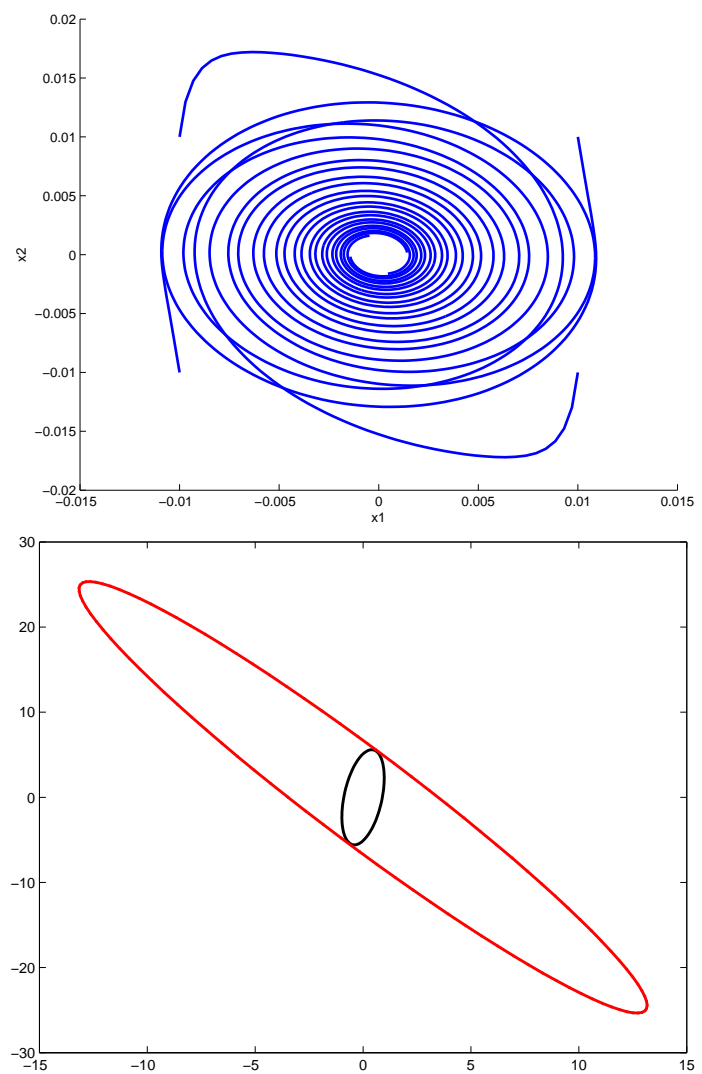

Fig. 6. Top: time evolution of $x_{1}$ and $x_{2}$ for sufficiently small initial conditions. Down: projection of the ellipsoids involved in (30) on the plane $\left(x_{3}, x_{4}\right)$.

$\delta$. Since $\delta<1$ we have $q^{0}=0$. It follows from the definition of $\mathcal{D}_{0}$, that the initial condition is not in $\mathcal{D}_{0}$. Thus, by considering the first $n+l_{0}$ components of the trajectory $(x, \zeta)$, we get that $\left(x, \zeta_{0}\right)$ is a trajectory of (4) on $[0, \bar{t}) \times\{0\}$ for a positive $\bar{t}$. Moreover from (37), all such maximal trajectories $(x, \zeta)$ do not return in $\mathcal{D}$, and their domains is of the form $[0, T) \times\{0\}$ with $T \in \mathbb{R}_{>0} \cup\{\infty\}$.

If $T<\infty$, then $(x, \zeta)$ eventually leaves any compact subset of $\mathbb{R}^{n} \times \mathbb{R}^{l}$. Since the system (12) with $u=\alpha_{0}\left(h(x), \zeta_{0}\right)$ is ISS, $z_{1}$ cannot grow unbounded if the $\left(x, \zeta_{0}\right)$ is bounded. Moreover the $z_{0}$-component is bounded. Therefore $\left(x(., 0), \zeta_{0}(., 0)\right)$ is a trajectory of (4) on $[0, T)$ starting from $\left\{\left(x, \zeta_{0}\right): V_{0}\left(x, \zeta_{0}\right) \leq\right.$ $\left.\varepsilon_{0 a}\right\}$, which grows unbounded. This contradicts item 1 of Assumption 2.

Therefore $T=\infty$ and $\left(x(., 0), \zeta_{0}(., 0), z_{0}(., 0), z_{1}(., 0)\right)$ is a trajectory of (4), (10) and (12) (with $u=\alpha_{0}\left(h(x), \zeta_{0}\right)$ ) on $[0, \infty)$, and thus we have $|x(t)|+\left|\zeta_{0}(t)\right|+\left|z_{0}(t)\right|+\left|z_{1}(t)\right| \leq \varepsilon$, for all $t \geq 0$. Also for all $t \geq 0, q(t)=0$. This is the local stability.

Moreover, due to the expression of the set $\mathcal{C}$, and due to (10), every trajectory of (6) starting at $\left(x^{0}, \zeta^{0}\right) \in \mathbb{R}^{n} \times(C \cup \mathcal{D})$, with $\omega\left(x^{0}, \zeta^{0}\right) \leq r$, has the hybrid time domain $[0, \infty) \times\{0\}$, $(x(t, 0), M \zeta(t, 0))=\left(x(t), \zeta_{0}(t)\right)$ and $\left(x, \zeta_{0}\right)$ is a trajectory to (4).

Let us establish global convergence. Let $\left(x^{0}, \zeta^{0}\right) \in \mathbb{R}^{n} \times$ $(\mathcal{L} \cup \mathcal{D})$ and $(x, \zeta)$ be a maximal trajectory of (6) defined on $\operatorname{dom}(x, \zeta)$ and starting at $\left(x^{0}, \zeta^{0}\right)$. First we prove that the discrete variable has to take the value 0 for some suitable 
hybrid time:

Claim 6.1: There exists $(t, j) \in \operatorname{dom}(x, \zeta)$, such that $q(t, j)=0$.

Proof of Claim 6.1. Let us prove this claim by contradiction and assume that

$$
q(t, j)=1, \forall(t, j) \in \operatorname{dom}(x, \zeta),
$$

then, $\operatorname{dom}(x, \zeta)=[0, T) \times\{0\}$, with $T \in \mathbb{R}_{>0} \cup\{\infty\}$. Moreover, $\left(x(., 0), \zeta_{1}(., 0)\right)$ is a trajectory of (5). If $T<\infty$, then $(x, \zeta)$ eventually leaves any compact subset of $\mathbb{R}^{n} \times \mathbb{R}^{l}$. Due to the expression of $\mathcal{C}_{1}$, we note that the $\zeta_{0}$-component and the $z_{0^{-}}$ component remain equal to 0 . Since the system (12) with $u=$ $\alpha_{1}\left(h(x), \zeta_{1}\right)$ is ISS, $z_{1}$ cannot grow unbounded if the $\left(x, \zeta_{1}\right)$ is bounded. Therefore $\left(x(., 0), \zeta_{1}(., 0)\right)$ is a trajectory of (5) which grows unbounded. This cannot occur due to item 3 of Assumption 2.

Therefore $T=\infty$, and $\left(x(., 0), \zeta_{1}(., 0)\right)$ is a trajectory of (5) defined on $[0, \infty)$, and according to item 3 of Assumption 2, there exists $\bar{t}>0$, such that we have, for all $t \geq \bar{t}, V_{1}(x(t, 0))+$ $\left|\zeta_{1}(t, 0)\right|<\varepsilon_{2}$. Moreover, from (12), we compute $\left|z_{1}(t, 0)\right| \leq$ $\left|z_{1}(\bar{t}, 0)\right| e^{-t+\bar{t}}+\max _{s \in[\bar{t}, t]} \rho_{1}\left(\alpha_{1}(h(x(s, 0))\right.$,

$\left.\left.\zeta_{1}(s, 0)\right), h(x(s, 0))\right)$. Using item 6 of Assumption 2, we get the existence of $\widetilde{t} \geq \bar{t}$ such that $z_{1}(\widetilde{t})<\varepsilon_{1 a}$. This contradicts (38), and concludes the proof of Claim 6.1.

Now, we prove that if the discrete variable is equal to 0 for all sufficiently large time, then the trajectory converges to the origin:

Claim 6.2: If there exists a hybrid time $(\bar{t}, \bar{j}) \in \operatorname{dom}(x, \zeta)$, such that, $q(t, j)=0$, for all $(t, j) \in \operatorname{dom}(x, \zeta),(t, j) \geq(\bar{t}, \bar{j})$, then the trajectory is complete and converges to the origin.

Proof of Claim 6.2. First we note that if there is not any jump after $(\bar{t}, \bar{j})$, then for all $(t, j) \in \operatorname{dom}(x, \zeta),(t, j) \geq(\bar{t}, \bar{j})$, we have $j=\bar{j}$, and due to the expression of $w$, for all $(t, \bar{j}) \in$ $\operatorname{dom}(x, \zeta), t \geq \bar{t}$, we have $\zeta(t, j) \notin \mathcal{D}$. Moreover $z_{0}(t, \bar{j}) \leq \varepsilon_{0 a}$ for such $(t, \bar{j})$ and $\left(x(., \bar{j}), \zeta_{0}(., \bar{j})\right)$ is a trajectory of (4) on the domain $\{t,(t, \bar{j}) \in \operatorname{dom}(x, \zeta), t \geq \bar{t}\}$.

Let us prove that the trajectory is complete. Let us assume the converse. Then the trajectory grows unbounded. The $z_{0}$ component and the $\zeta_{1}$-component are bounded due to the expression of $\mathcal{C}_{0}$ and since $q(t, \bar{j})=0$, for all $(t, \bar{j}) \in \operatorname{dom}(x, \zeta)$, $t \geq \bar{t}$. Since the system (12) with $u=\alpha_{0}\left(h(x), \zeta_{0}\right)$ is ISS, $z_{1}$ cannot grow unbounded if the $\left(x, \zeta_{0}\right)$ is bounded. Therefore we get a trajectory of (4) which is unbounded. With item 2 of Assumption 2 this implies that the $z_{0}$ grows also unbounded, which is a contradiction with the expression of $\mathcal{C}_{0}$. This contradiction implies that the trajectory is complete.

Recall that $\left(x, \zeta_{0}, z_{0}\right)(., \bar{j})$ is a maximal trajectory of (4) and (10) on $[\bar{t}, \infty)$. From item 2 of Assumption 2, (11), $\varepsilon_{0 a}<\varepsilon_{0 b}$, and the fact that $\beta_{0}$ is a function of class $\mathcal{K} \mathcal{L}, \bar{t}$ may be assumed to be such that, for all $t$ in $[\bar{t}, \infty)$, the inequality $V_{0}\left(x(t, \bar{j}), \zeta_{0}(t, \bar{j})\right) \leq \varepsilon_{0 b}$ holds. But, according to item 1 of Assumption 2, this set belongs to the basin of attraction of the target set. Hence, the $x$ - and $\zeta_{0}$-components of the trajectory converge to 0 . Due to the expression of $\mathcal{C}_{0}$, the $\zeta_{1}$-component is equal to 0 , and since the systems (10) and (12) (with $\left.u=\alpha_{0}\left(h(x), \zeta_{0}\right)\right)$ are ISS, the $\left(z_{0}, z_{1}\right)$-component tends also to zero. This concludes the proof of Claim 6.2.
In the last intermediate result, the discrete variable cannot switch back and forth between $q=0$ and $q=1$ :

Claim 6.3: There does not exist a non decreasing sequence of hybrid times $\left(\left(t_{n}, j_{n}\right)_{n \in \mathbb{N}}\right) \in \operatorname{dom}(x, \zeta)$, such that we have, for all $n \in \mathbb{N}$,

$$
q\left(t_{2 n}, j_{2 n}\right)=0, q\left(t_{2 n+1}, j_{2 n+1}\right)=1 .
$$

Proof of Claim 6.3. First we note that instantaneous Zeno trajectories ${ }^{6}$ are impossible due to the expressions of $\mathcal{D}_{0}$ and $\mathcal{D}_{1}$. Let us prove Claim 6.3 by contradiction and assume that there exists a non decreasing sequence of hybrid times $\left(\left(t_{n}, j_{n}\right)_{n \in \mathbb{N}}\right) \in \operatorname{dom}(x, z)$, such that we have (39) for all $n \in \mathbb{N}$. Without loss of generality we may assume that we have no jump between two points of this sequence and that $j_{n}=n$. Due to the expression of the function $w$, and the sets $C$ and $\mathcal{D}$, for all $n \in \mathbb{N}$, we have to flow in $\mathcal{C}_{0}$ between $\left(t_{2 n}, 2 n\right)$ and $\left(t_{2 n+1}, 2 n\right)$, and we have to flow in $C_{1}$ between $\left(t_{2 n+1}, 2 n+1\right)$ and $\left(t_{2 n+2}, 2 n+1\right)$. Note that $\zeta\left(t_{2 n+2}, 2 n+1\right) \in$ $\mathcal{D}_{1}$ and thus $z_{1}\left(t_{2 n+2}, 2 n+1\right) \leq \varepsilon_{1 a}$, for all $n \in \mathbb{N}$. Therefore, with item 4 of Assumption 2, there exists $N>0$ such that we have $V_{1}\left(x\left(t_{2 N+2}, 2 N+2\right)\right)=V_{1}\left(x\left(t_{2 N+2}, 2 N+1\right)\right) \leq \varepsilon_{1 b}$. Thus, between $\left(t_{2 N+2}, 2 N+2\right)$ and $\left(t_{2 N+3}, 2 N+2\right),\left(x, \zeta_{0}\right)$ is a trajectory of (4) starting from $\left\{\left(x, \zeta_{0}\right), V_{1}(x) \leq \varepsilon_{1 b}, \zeta_{0}=0\right\}$. It follows from item 5 of Assumption 2, that $\rho_{0}\left(h\left(x\left(t_{2 N+3}, 2 N+\right.\right.\right.$ 2)), $\left.\zeta_{0}\left(t_{2 N+3}, 2 N+2\right)\right)<\varepsilon_{0 a}$. Thus with (10), $z_{0}\left(t_{2 N+3}, 2 N+\right.$ $2)<\varepsilon_{0 a}$. This contradicts $\zeta\left(t_{2 N+3}, 2 N+2\right) \in \mathcal{D}_{0}$ and concludes the proof of Claim 6.3.

Let us combine the previous claims. First, due to Claim 6.1 , there exists $(t, j) \in \operatorname{dom}(x, \zeta)$ such that $q(t, j)=0$, and there does not exist a hybrid time $\left(t^{\star}, j^{\star}\right) \in \operatorname{dom}(x, \zeta)$, such that, $q(t, j)=1$, for all $(t, j) \in \operatorname{dom}(x, \zeta),(t, j) \geq\left(t^{\star}, j^{\star}\right)$. Now with Claim 6.3, there exists $(\bar{t}, \bar{j}) \in \operatorname{dom}(x, \zeta)$, such that the assumption of Claim 6.2 holds. Thus the trajectory is complete and tends to the origin. Therefore the origin is asymptotically stable for the system (6).

All what remains to establish is the robustness issue. It follows mainly from [23, Theo. 4.3]. To check that point, let us introduce the closed subsets of $\mathbb{R}^{n} \times \mathbb{R}^{l_{0}} \times \mathbb{R}^{l_{1}} \times \mathbb{R}_{>0} \times \mathbb{R}_{>0}$

$$
\begin{aligned}
\mathfrak{C}_{q} & =\left\{\left(x, \zeta_{0}, \zeta_{1}, z_{0}, z_{1}\right), \text { s.t. }\left(\zeta_{0}, \zeta_{1}, z_{0}, z_{1}, q\right) \in \mathcal{C}_{q}\right\}, \\
\mathfrak{D}_{q} & =\left\{\left(x, \zeta_{0}, \zeta_{1}, z_{0}, z_{1}\right), \text { s.t. }\left(\zeta_{0}, \zeta_{1}, z_{0}, z_{1}, q\right) \in \mathcal{D}_{q}\right\} .
\end{aligned}
$$

The hybrid system (6) can be rewritten as $(\dot{x}, \dot{\xi})=$ $F_{q}(x, \xi)$ if $(x, \xi) \in \mathfrak{C}_{q}, q^{+}=G_{q}(x, \xi)$ if $(x, \xi) \in \mathfrak{D}_{q}$ with obvious definitions for the functions $F_{q}: \mathfrak{C}_{q} \rightarrow \mathbb{R}^{n} \times \mathbb{R}^{l_{0}} \times \mathbb{R}^{l_{1}} \times$ $\mathbb{R}_{\geq 0} \times \mathbb{R}_{\geq 0}$, and $G_{q}: \mathfrak{D}_{q} \rightarrow \mathbb{R}^{n} \times \mathbb{R}^{l_{0}} \times \mathbb{R}^{l_{1}} \times \mathbb{R}_{\geq 0} \times \mathbb{R}_{\geq 0}$. The functions $h, \alpha_{0}, \alpha_{1}, F_{q}$, and $G_{q}$ are continuous on their domains of definition. Now the family $\left\{\mathfrak{C}_{q}\right\}_{q \in\{0,1\}}$ forms a covering of $\mathbb{R}^{n} \times \mathbb{R}^{l_{0}} \times \mathbb{R}^{l_{1}} \times \mathbb{R}_{\geq 0} \times \mathbb{R}_{\geq 0}$, and for all $q \in\{0,1\}$, we have $\mathfrak{C}_{q} \cup \mathfrak{D}_{q}=\mathbb{R}^{n} \times \mathbb{R}^{l_{0}} \times \mathbb{R}^{l_{1}} \times \mathbb{R}_{\geq 0} \times \mathbb{R}_{\geq 0}$. Therefore we can apply [23, Theo. 4.3], and we have the robustness of the asymptotic stability as in Theorem 3.2.

Proof of Theorem 2.1. The proof of Theorem 2.1 follows from Claim 3.1 and Theorem 3.2.

\footnotetext{
${ }^{6}$ We recall that a Zeno trajectory is said to be instantaneous, if the time $t$ is eventually constant.
} 


\section{Proof of Proposition 4.2 AND Theorem 4.4}

Proof of Proposition 4.2. We assume that Assumption 3 is satisfied. Let us state the items of Assumption 4 successively.

Due to item 1 of Assumption 3, the origin of linear system (3) is asymptotically stable and thus (see [25, Chap. 7, Theo. 32]), the pair $(C, A)$ is detectable. Therefore there exist a symmetric positive definite matrix $P_{1} \in \mathbb{R}^{n \times n}$, and a matrix $L \in \mathbb{R}^{n \times p}$ such that inequality (22) holds.

Moreover, since the origin of the system (2) is locally asymptotically stable, there exists a symmetric positive definite matrix $P_{0}$ and a positive value $\varepsilon_{3}$ such that item 1 of Assumption 4 holds. We easily see that there exist a symmetric positive definite matrix $Q_{0} \in \mathbb{R}^{l_{0} \times l_{0}}$ and a sufficiently small positive value $\varepsilon_{0 b}$ such that (24) holds. Now observe that there exist a matrix $H \in \mathbb{R}^{m \times\left(n+l_{0}\right)}$ and a sufficiently small positive value $\varepsilon_{1 b}$ such that (23) and (25) hold (the value of $\varepsilon_{1 b}$ may be reduced). Moreover, using Schur complements, we check that there exist a symmetric positive definite matrix $P \in \mathbb{R}^{m \times m}$, a symmetric positive semidefinite matrix $N \in \mathbb{R}^{\left(m+p+l_{0}\right) \times\left(m+p+l_{0}\right)}$, and sufficiently small positive values $\varepsilon_{1 a}<\varepsilon_{1 b}$ such that the conditions (26) and (27) hold. Thus item 4 of Assumption 4 holds.

By continuity of $\rho_{1}$ and $\rho_{1}(0,0)=0$, and using item 2 of Assumption 3, there exists $\varepsilon_{2}>0$ such that item 3 of Assumption 4 holds. Using item 2 of Assumption 3 again, item 2 of Assumption 4 is satisfied. Therefore we get that Assumption 4 holds if Assumption 3 is satisfied, as claimed in Proposition 4.2.

Let us state that following Algorithm 4.3, we compute all the variables allowing to define the hybrid controller (16). Due to item 1 of Assumption 3, the pair $(C, A)$ is detectable, and there exist a symmetric positive definite matrix $P_{1} \in \mathbb{R}^{n \times n}$, and a matrix $\mathcal{L} \in \mathbb{R}^{n \times p}$ such that $P_{1} A+\mathcal{L} C+A^{\prime} P_{1}+\mathcal{L} C^{\prime} \leq-2 P_{1}$. Therefore, by letting $L=L_{1}^{-1} \mathcal{L}$ as done in step 1 of Algorithm 4.3 , the inequality (22) follows.

The determination of the exact basin of attraction of (20) is in general impossible; however, as soon as $\mathbf{A}+\mathbf{B} K$ is Hurwitz, there exist many relaxations, and convex problems written in terms of LMI, to compute a quadratic Lyapunov function for (20) and a convex approximation of the basin of attraction of (20) (see e.g., [13], [28]). One possible approach is to use a modified sector condition as introduced in [8] (see also [14]). Denoting $\mathbf{x}=\left[x^{\prime} \zeta_{0}^{\prime}\right]^{\prime}$, it is suggested in [8] a convex problem to compute a quadratic Lyapunov function $\mathbf{x} \mapsto \mathbf{x} P_{0} \mathbf{x}$ for (20):

Proposition 7.1: ([8]) If there exist a symmetric positive definite matrix $W_{0}$ in $\mathbb{R}^{\left(n+l_{0}\right) \times\left(n+l_{0}\right)}$, a matrix $Z \in \mathbb{R}^{m \times\left(n+l_{0}\right)}$ and a diagonal positive matrix $S \in \mathbb{R}^{\left(n+l_{0}\right) \times\left(n+l_{0}\right)}$ such that

$$
\left[\begin{array}{cc}
W_{0} \mathscr{A}^{\prime}+\mathcal{A} W_{0} & \mathbf{B} S-Z^{\prime} \\
\star & -2 S
\end{array}\right]<0,
$$

and

$$
\left[\begin{array}{cc}
W_{0} & W_{0} K_{(i)}^{\prime}-Z_{(i)}^{\prime} \\
\star & \bar{u}_{(i)}^{2}
\end{array}\right] \geq 0, i=1, \ldots, m,
$$

where $\mathcal{A}=\mathbf{A}+\mathbf{B} K$, and $K_{(i)}\left(\right.$ resp. $\left.Z_{(i)}\right)$ denotes the ith row of $K$ (resp. Z), then, denoting $P_{0}=W_{0}^{-1}$, the ellipsoid $\{\mathbf{x} \in$ $\left.\mathbb{R}^{n+l_{0}}, \mathbf{x}^{\prime} P_{0} \mathbf{x} \leq 1\right\}$ is included in the basin of attraction of (20), and the function $\mathbf{x} \mapsto \mathbf{x}^{\prime} P_{0} \mathbf{x}$ decreases along the trajectories of (20), when starting in $\left\{\mathbf{x} \in \mathbb{R}^{n+l_{0}}, \mathbf{x}^{\prime} P_{0} \mathbf{x} \leq 1\right\}$.

Letting $\varepsilon_{3}=1$ we get that $P_{0}$ and $\varepsilon_{3}$ satisfy item 1 of Assumption 4. Now we compute a symmetric positive matrix $R$ and a positive value $\varepsilon$ solution of (30). We get that the system (20) is asymptotically stable in $\left\{\mathbf{x}, \mathbf{x}^{\prime} \operatorname{diag}\left(P_{1}, Q_{0}\right) \mathbf{x} \leq \varepsilon_{0 b}\right\}$.

Given $0<\varepsilon_{0 a}<\varepsilon_{0 b}$, we compute a matrix $H \in \mathbb{R}^{m \times\left(n+l_{0}\right)}$ and a positive value $\hat{\varepsilon}$ satisfying (31). Then we compute a symmetric positive definite matrix $P \in \mathbb{R}^{m \times m}$, a symmetric positive semidefinite matrix $N \in \mathbb{R}^{\left(m+p+l_{0}\right) \times\left(m+p+l_{0}\right)}$ and a positive value $\widetilde{\varepsilon}$ satisfying (26) and (32). Thus the computed variables are such that we have item 4 of Assumption 4 . Letting $0<\varepsilon_{1 a}<\varepsilon_{1 b}$, by item 2 of Assumption 3, there exists $0<\varepsilon_{2}$ such that item 2 and item 3 of Assumption 4 hold. Therefore following Algorithm 4.3, we compute all the variables allowing to define the hybrid controller (16) by solving only LMIs. This concludes the proof of Proposition 4.2.

Proof of Theorem 4.4. Let Assumption 4 hold, and consider the system (2) in closed loop with the hybrid feedback (16). Recalling Theorem 3.2, to state Theorem 4.4 it is sufficient to prove the following:

Claim 7.2: Assumption 2 holds if Assumption 4 is satisfied.

Proof of Claim 7.2. We state the items of Assumption 2 successively. Let us construct an IOSS-Lyapunov function for (2). With (22) in Assumption 4, and letting $V_{1}^{\circ}=\nabla V_{1}(x) \cdot(A x+$ $B u$ ), we compute

$$
\begin{aligned}
\stackrel{\circ}{V}_{1}= & 2 x^{\prime} P_{1}(A x+B u), \\
= & 2 x^{\prime} P_{1}(A+L C) x+2 x^{\prime} P_{1} B u-2 x^{\prime} P_{1} L y, \\
\leq & -2 V_{1}(x)+2 x^{\prime} P_{1} B u-2 x^{\prime} P_{1} L y, \\
\leq & -2 V_{1}(x)+\frac{1}{2} V_{1}(x)+2 u^{\prime} B^{\prime} P_{1} B u+\frac{1}{2} V_{1}(x) \\
& +2 y^{\prime} L^{\prime} P_{1} L y \\
\leq & -V_{1}(x)+2\left[\begin{array}{l}
u \\
y
\end{array}\right]^{\prime}\left[\begin{array}{cc}
B^{\prime} P_{1} B & 0 \\
\star & L^{\prime} P_{1} L
\end{array}\right]\left[\begin{array}{l}
u \\
y
\end{array}\right] .
\end{aligned}
$$

Therefore we get the existence of a function $\beta_{1}$ of class $\mathcal{K} \mathcal{L}$ such that item 4 of Assumption 2 holds. Now, defining $V_{0}$ : $\mathbb{R}^{n} \times \mathbb{R}^{I_{0}} \rightarrow \mathbb{R}_{\geq 0}$ with $V_{0}\left(x, \zeta_{0}\right)=x^{\prime} P_{1} x+\zeta_{0}^{\prime} Q_{0} \zeta_{0}$, and letting $\stackrel{\circ}{V}_{0}=\nabla V_{0}\left(x, \zeta_{0}\right) \cdot\left(A x+B \operatorname{sat}\left(C_{0} \zeta_{0}+D_{0} C x\right), A_{0} \zeta_{0}+B_{0} C x\right)$, we compute with (40)

$$
\begin{aligned}
\stackrel{\circ}{V}_{0} \leq & -V_{1}(x)+2\left[\begin{array}{c}
\operatorname{sat}\left(C_{0} \zeta_{0}+D_{0} y\right) \\
y
\end{array}\right]^{\prime}\left[\begin{array}{cc}
B^{\prime} P_{1} B & 0 \\
\star & L^{\prime} P_{1} L
\end{array}\right] \\
& \times\left[\begin{array}{c}
\operatorname{sat}\left(C_{0} \zeta_{0}+D_{0} y\right) \\
y
\end{array}\right]+2 \zeta_{0}^{\prime} Q_{0}\left(A_{0} \zeta_{0}+B_{0} y\right), \\
\leq & -V_{1}(x)+2 \zeta_{0}^{\prime} Q_{0}\left(A_{0} \zeta_{0}+B_{0} y\right) \\
& +4\left(\operatorname{sat}\left(C_{0} \zeta_{0}+D_{0} y\right)-C_{0} \zeta_{0}-D_{0} y\right)^{\prime} B^{\prime} P_{1} B\left(C_{0} \zeta_{0}+D_{0} y\right) \\
& +2\left[\begin{array}{c}
\operatorname{sat}\left(C_{0} \zeta_{0}+D_{0} y\right)-C_{0} \zeta_{0}-D_{0} y \\
y
\end{array}\right]^{\prime} \\
& \times\left[\begin{array}{cc}
B^{\prime} P_{1} B & 0 \\
\star & L^{\prime} P_{1} L
\end{array}\right]\left[\begin{array}{c}
\operatorname{sat}\left(C_{0} \zeta_{0}+D_{0} y\right)-C_{0} \zeta_{0}-D_{0} y \\
y
\end{array}\right] \\
& +2\left(C_{0} \zeta_{0}+D_{0} y\right)^{\prime} B^{\prime} P_{1} B\left(C_{0} \zeta_{0}+D_{0} y\right),
\end{aligned}
$$


and thus

$$
\begin{aligned}
{\stackrel{\circ}{V_{0}} \leq} & -V_{0}(x)+\left[\begin{array}{c}
\operatorname{sat}\left(C_{0} \zeta_{0}+D_{0} y\right)-C_{0} \zeta_{0}-D_{0} y \\
y \\
\zeta_{0}
\end{array}\right] \\
& \times N\left[\begin{array}{c}
\operatorname{sat}\left(C_{0} \zeta_{0}+D_{0} y\right)-C_{0} \zeta_{0}-D_{0} y \\
y \\
\zeta_{0}
\end{array}\right]
\end{aligned}
$$

where $N$ satisfies (26). Therefore, by defining $\rho_{0}$ as in Theorem 4.4, we get the existence of a function $\beta_{0}$ of class $\mathcal{K} \mathcal{L}$ such that we have item 2 of Assumption 2. Item 1 of Assumption 2 follows from item 1 of Assumption 4, (23) and (24). The following remark will be useful.

Remark 7.3: One way to have item 5 of Assumption 2 is to assume the existence of a function $\alpha: \mathbb{R}_{\geq 0} \rightarrow \mathbb{R}_{\geq 0}$ of class $\mathcal{K}_{\infty}$ such that

1. we have $\left(x^{\prime}, 0\right) P_{0}\left(x^{\prime}, 0\right)^{\prime} \leq \alpha\left(V_{1}(x)\right)$ for all $x$ such that $V_{1}(x) \leq \varepsilon_{1 b}$

2. for each trajectory of (20) starting from $\left\{\left(x, \zeta_{0}\right), \quad\left(x^{\prime}, \zeta_{0}^{\prime}\right) P_{0}\left(x^{\prime}, \zeta_{0}^{\prime}\right)^{\prime} \leq \alpha\left(\varepsilon_{1 b}\right)\right\}$, we have $\rho_{0}\left(C x(t), \zeta_{0}(t)\right)<\varepsilon_{0 a}$, for all $t \geq 0$.

Note that, due to (24), we have $\left(x^{\prime}, 0\right) P_{0}\left(x^{\prime}, 0\right)^{\prime} \leq \frac{1}{\varepsilon_{0 b}} V_{1}(x)$, for all $x \in \mathbb{R}^{n}$. Thus, with Remark 7.3, (23) and item 1 of Assumption 4, item 5 of Assumption 2 is satisfied as soon as $\rho_{0}\left(C x, \zeta_{0}\right)<\varepsilon_{0 a}$, for all $\left(x, \zeta_{0}\right) \in \mathbb{R}^{n} \times \mathbb{R}^{l_{0}}$ such that $\left(x^{\prime}, \zeta_{0}^{\prime}\right) P_{0}\left(x^{\prime}, \zeta_{0}^{\prime}\right)^{\prime} \leq \frac{\varepsilon_{1 b}}{\varepsilon_{0 b}}$. Therefore, to prove that item 5 of Assumption 2 holds, it is sufficient to state that, for all $\mathbf{x} \in \mathbb{R}^{n+l_{0}} \backslash\{0\}$, such that $\mathbf{x}^{\prime} P_{0} \mathbf{x} \leq \frac{\varepsilon_{1 b}}{\varepsilon_{0 b}}$, we have

$$
\left[\begin{array}{c}
\operatorname{sat}(K \mathbf{x})-K \mathbf{x} \\
\mathbf{x}
\end{array}\right]^{\prime} \widetilde{N}\left[\begin{array}{c}
\operatorname{sat}(K \mathbf{x})-K \mathbf{x} \\
\mathbf{x}
\end{array}\right]<\frac{\varepsilon_{0 a} \varepsilon_{0 b}}{\varepsilon_{1 b}} \mathbf{x}^{\prime} P_{0} \mathbf{x},
$$

where $\widetilde{N}=\operatorname{diag}\left(I_{p}, C^{\prime}, I_{l_{0}}\right) N \operatorname{diag}\left(I_{p}, C, I_{l_{0}}\right)$. Let us note that, applying Schur complements, the condition (25) is equivalent to $\mathbf{x}^{\prime} H_{(i)}^{\prime} \bar{u}_{i} \bar{u}_{i} H_{(i)} \mathbf{x} \leq \frac{\varepsilon_{0 b}}{\varepsilon_{1 b}} \mathbf{x}^{\prime} P_{0} \mathbf{x}$, and, using the fact that the saturation level is decentralized, for all $\mathbf{x} \in \mathbb{R}^{n+l_{0}}$, satisfying $\mathbf{x}^{\prime} P_{0} \mathbf{x} \leq \frac{\varepsilon_{1 b}}{\varepsilon_{0 b}}$, we have $\operatorname{sat}(H \mathbf{x})=H \mathbf{x}$. Now, for each symmetric positive definite matrix $P \in \mathbb{R}^{m \times m}$, denoting $\mathbf{u}=K \mathbf{x}$, we get, for all $\mathbf{x} \in \mathbb{R}^{n \times l_{0}}$ such that $\mathbf{x}^{\prime} P_{0} \mathbf{x} \leq \frac{\varepsilon_{1 b}}{\varepsilon_{0 b}}$,

$$
(\mathbf{u}-\operatorname{sat}(\mathbf{u}))^{\prime} P(\operatorname{sat}(\mathbf{u})-H \mathbf{x}) \geq 0 .
$$

Indeed, consider this inequality for each saturation function one at a time. If $\mathbf{u}_{(i)}=\operatorname{sat}_{(i)}\left(\mathbf{u}_{(i)}\right)$ then there is nothing to check. If $\mathbf{u}_{(i)} \neq \operatorname{sat}_{(i)}\left(\mathbf{u}_{(i)}\right)$ then $\mathbf{u}_{(i)}-\operatorname{sat}\left(\mathbf{u}_{(i)}\right)$ has the same sign as $\operatorname{sat}\left(\mathbf{u}_{(i)}\right)-H_{(i)} \mathbf{x}$ since $H_{(i)} \mathbf{x}=\operatorname{sat}_{(i)}\left(H_{(i)} \mathbf{x}\right)$ where $H_{(i)}$ denotes the $i$ th row of $H$.

From (42), for all $\mathbf{x} \in \mathbb{R}^{n \times l_{0}}$ such that $\mathbf{x}^{\prime} P_{0} \mathbf{x} \leq$ $\frac{\varepsilon_{1 b}}{\varepsilon_{0 b}}$, we have $(\mathbf{u}-\operatorname{sat}(\mathbf{u}))^{\prime} P(\operatorname{sat}(\mathbf{u})-\mathbf{u}+K \mathbf{x}-H \mathbf{x}) \geq$ 0, i.e. $\left[\begin{array}{c}\operatorname{sat}(\mathbf{u})-\mathbf{u} \\ \mathbf{x}\end{array}\right]^{\prime}\left[\begin{array}{cc}2 P & P(K-H) \\ \star & 0\end{array}\right]\left[\begin{array}{c}\operatorname{sat}(\mathbf{u})-\mathbf{u} \\ \mathbf{x}\end{array}\right] \leq 0$. Thus, using the $\mathrm{S}$ procedure, the condition (41) is implied by $\widetilde{N}-\frac{\varepsilon_{0 a} \varepsilon_{0 b}}{\varepsilon_{1 b}} \operatorname{diag}\left(0, P_{0}\right)-\left[\begin{array}{cc}2 P & P(K-H) \\ \star & 0\end{array}\right]<0$. We get that (27) implies (41), and thus we have item 5 of Assumption 2.

Due to item 2 (resp. item 3) of Assumption 4, we have item 3 (resp. item 6) of Assumption 2. This concludes the proof of Claim 7.2, and, with Theorem 3.2, the proof of Theorem 4.4.

\section{CONCLUSION}

Given two stabilizing output feedbacks controllers (one being globally asymptotically stabilizing, the other one being only locally asymptotically stabilizing) for the nonlinear control system (1), we designed a hybrid output feedback controller that is equal to the local controller on a neighborhood of the origin, and that is globally asymptotically stabilizing. To solve this uniting problem, we assumed that the nonlinear control system is IOSS.

We have also considered linear systems with saturation at the input. Given two stabilizing output feedbacks controllers (one being a linear but only locally asymptotically stabilizing, the other being nonlinear and globally attractive), we numerically computed a hybrid output feedback controller that is equal to the local controller for initial condition in a neighborhood of the origin, and that is globally asymptotically stabilizing.

Combining [23, Theorem 4.3] and the regularity of the data of our hybrid feedback, we achieved the robustness with respect to (small) measurement noise, actuator errors and external disturbances. We noted that the uniting problem may not have a solution in terms of classical (continuous or discontinuous) controllers as explained in Remark 2.2. The main results and the robustness were illustrated on simulations.

\section{REFERENCES}

[1] V. Andrieu and L. Praly. A unifying point of view on output feedback designs for global asymptotic stabilization. Automatica, 45(8):17891798, 2009.

[2] D. Angeli, E.D. Sontag, and Y. Wang. A characterization of integral input-to-state stability. IEEE Transactions on Automatic Control, 45(6):1082-1097, 2000.

[3] J.-P. Aubin, J. Lygeros, M. Quincampoix, S. Sastry, and N. Seube. Impulse differential inclusions: a viability approach to hybrid systems. IEEE Trans. Automat. Control, 47(1):2-20, 2002.

[4] S. Battilotti. Sufficient conditions for global output regulation of nonlinear interconnected systems. Automatica, 35(5):829-835, 1999.

[5] Y.-Y. Cao, Z. Lin, and D.G. Ward. An antiwindup approach to enlarging domain of attraction for linear systems subject to actuator saturation. IEEE Transactions on Automatic Control, 47(1):140-145, 2002.

[6] F.H. Clarke, Y.S. Ledyaev, L. Rifford, and R.J. Stern. Feedback stabilization and Lyapunov functions. SIAM J. Control Optim., 39(1):2548, 2000.

[7] F.H. Clarke, Y.S. Ledyaev, and R.J. Stern. Asymptotic stability and smooth Lyapunov functions. J. Diff. Eq., 149(1):69-114, 1998.

[8] J.M. Gomes da Silva Jr and S. Tarbouriech. Antiwindup design with guaranteed regions of stability : an LMI-based approach. IEEE Transactions on Automatic Control, 50:106-111, 2005.

[9] D.V. Efimov. Uniting global and local controllers under acting disturbances. Automatica, 42(3):489-495, 2006.

[10] R. Goebel, J. Hespanha, A.R. Teel, C. Cai, and R. Sanfelice. Hybrid systems: generalized solutions and robust stability. In IFAC Symp. on Nonlinear Control Systems (NOLCOS), pages 1-12, Stuttgart, Germany, 2004.

[11] R. Goebel and A.R. Teel. Solutions to hybrid inclusions via set and graphical convergence with stability theory applications. Automatica, 42(4):573-587, 2006.

[12] G. Grimm, J. Hatfield, I. Postlethwaite, A.R. Teel, M.C. Turner, and L. Zaccarian. Antiwindup for stable linear systems with input saturation: an LMI-based synthesis. IEEE Transactions on Automatic Control, 48(9):1509-1525, 2003.

[13] T. Hu and Z. Lin. Control systems with actuator saturation: analysis and design. Birkhauser, Boston, 2001.

[14] T. Hu, A.R. Teel, and L. Zaccarian. Stability and performance for saturated systems via quadratic and nonquadratic Lyapunov functions. IEEE Transactions on Automatic Control, 51(11):1770-1786, 2006. 
[15] G. Kaliora, A. Astolfi, and L. Praly. Norm estimators and global output feedback stabilization of nonlinear systems with iss inverse dynamics. IEEE Transactions on Automatic Control, 51(3):493-498, 2006.

[16] H.K. Khalil. Nonlinear Systems. Prentice-Hall, 3rd edition, 2002.

[17] M. Krichman, E.D. Sontag, and Y. Wang. Input-output-to-state stability. SIAM J. Control Optim., 39:1874-1928, 2001.

[18] Y.S. Ledyaev and E.D. Sontag. A Lyapunov characterization of robust stabilization. Nonlinear Analysis, 37:813-840, 1999.

[19] J. Lygeros, K.H. Johansson, S.N. Simić, J. Zhang, and S.S. Sastry. Dynamical properties of hybrid automata. IEEE Transactions on Automatic Control, 48(1):2-17, 2003.

[20] R. Marino and P. Tomei. A class of globally output feedback stabilizable nonlinear nonminimum phase systems. IEEE Transactions on Automatic Control, 50(12):2097-2101, 2005.

[21] E.F. Mulder, M.V. Kothare, and M. Morari. Multivariable antiwindup controller synthesis using linear matrix inequalities. Automatica, 37(9):1407-1416, 2001.

[22] C. Prieur. Uniting local and global controllers with robustness to vanishing noise. Math. Control Signals Systems, 14:143-172, 2001.

[23] C. Prieur, R. Goebel, and A.R. Teel. Hybrid feedback control and robust stabilization of nonlinear systems. IEEE Transactions on Automatic Control, 52(11):2103-2117, 2007.

[24] C. Prieur and L. Praly. A tentative direct Lyapunov design of output feedbacks. In IFAC: Symp. on Nonlinear Control Systems (NOLCOS), pages 1121-1126, Stuttgart, Germany, 2004.

[25] E. Sontag. Mathematical Control Theory: Deterministic Finite Dimensional Systems. Springer, New York, second edition, 1998.

[26] E.D. Sontag. Clocks and insensitivity to small measurement errors. ESAIM Control Optim. Calc. Var, 4:537-557, 1999.

[27] E.D. Sontag. Input to state stability: Basic concepts and results. In Nonlinear and Optimal Control Theory, pages 163-220. SpringerVerlag, Berlin, 2007.

[28] S. Tarbouriech, G. Garcia, and A.H. Glattfelder (eds.). Advanced strategies in control systems with input and output constraints. In $L N C S$, volume 346. Springer Verlag, 2007.

[29] A.R. Teel, O.E. Kaiser, and R.M. Murray. Uniting local and global controllers for the Caltech ducted fan. In American Control Conf., pages 1539-1543, Albuquerque NM, 1997.

[30] A.R. Teel and N. Kapoor. Uniting local and global controllers. In European Control Conf., Bruxelles, Belgium, 1997.

[31] L. Zaccarian and A.R Teel. Nonlinear scheduled anti-windup design for linear systems. IEEE Transactions on Automatic Control, 49(11):20552061, 2004.

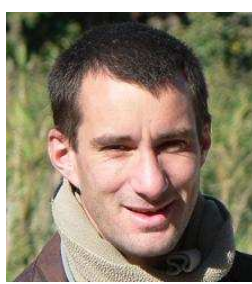

Christophe Prieur graduated in Mathematics from the Ecole Normale Supérieure de Cachan, France in 2000. He received the Ph.D degree in 2001 in Applied Mathematics from the Université ParisSud, France. From 2002 he is an associate researcher CNRS at the laboratory SATIE, Ecole Normale Supérieure de Cachan, at the LAAS, Toulouse (2004-2010), and from 2010 at the Gipsa-lab, Grenoble, France. His current research interests include nonlinear control theory, hybrid systems, and control of nonlinear partial differential equations.

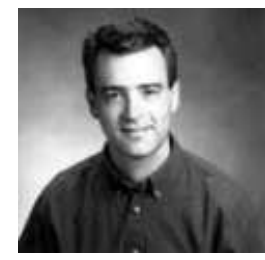

Andrew R. Teel received his A.B. degree in Engineering Sciences from Dartmouth College in Hanover, New Hampshire, in 1987, and his M.S. and Ph.D. degrees in Electrical Engineering from the University of California, Berkeley, in 1989 and 1992, respectively. After receiving his Ph.D., Dr. Teel was a postdoctoral fellow at the Ecole des Mines de Paris in Fontainebleau, France. In 1992 he joined the faculty of the Electrical Engineering Department at the University of Minnesota where he was an assistant professor. In 1997, Dr. Teel joined the faculty of the Electrical and Computer Engineering Department at the University of California, Santa Barbara, where he is currently a professor. 